\title{
Assessing moment-based boundary conditions for the lattice Boltzmann equation: a study of dipole-wall collisions
}

\author{
Seemaa Mohammed ${ }^{\mathrm{a}}$, David Graham ${ }^{\mathrm{a}}$, Tim Reis ${ }^{\mathrm{b}}$ \\ ${ }^{a}$ School of Computing Electronics and Mathematics, Plymouth University, PL4 8AA, UK \\ ${ }^{b}$ Department of Mathematical Science, University of Greenwich, SE10 9LS, UK
}

\begin{abstract}
The accuracy of moment-based boundary conditions for no slip walls in lattice Boltzmann simulations is examined numerically by using the dipole-wall collision benchmark test for both normal and oblique cases. In the normal case the dipole hits the wall perpendicularly while in the oblique case the dipole hits the wall at an angle of $30^{\circ}$ to the horizontal. Boundary conditions are specified precisely at grid points by imposing constraints upon hydrodynamic moments only. These constraints are then translated into conditions for the unknown lattice Boltzmann distribution functions at boundaries. The two relaxation time (TRT) model is used with a judiciously chosen product of the two relaxation times. Stable results are achieved for higher Reynolds number up to 10000 for the normal collision and up to 7500 for the oblique case. An excellent agreement with a benchmark data is observed and the local boundary condition implementation is shown to be second order accurate.
\end{abstract}

Keywords: lattice Boltzmann equation, moment-based boundary conditions, two relaxation times, dipole-wall collision

\section{Introduction}

Vortex dipole-wall collision in two dimensional flows is an important problem that has been the subject of numerous recent studies. In this flow two counter rotating vortices are propelled towards a solid boundary with which they collide. Interactions between dipoles and frictional boundaries are found in a lot of natural phenomena. For example, the effect of the ground on the formulation of the secondary vortices when an aeroplane takes off or lands is one of these problems [1]. Another phenomena is the formulation of the large scale vortices in geographical turbulence in the edges of coastal and oceans like the Black Sea [2]. Many laboratory experiments and numerical approximation methods have been used to study the monopole (one vortex) and the dipole (two vortex) flow. Orlandi [1] is one of the earliest researchers to study the wall dipole collision numerically and the effect of the flat solid walls on the rotating vortex. Coutsias and Lynove [3] employed Fourier-Chebyshev expansions with a spectral scheme to study the creation of the vortices from the interaction between the no slip walls and the dipole in a periodic channel. Clercx and Heijst

Email addresses: seemaa.mohammedeplymouth.ac.uk (Seemaa Mohammed), D. Grahameplymouth.ac.uk (David Graham), T.Reis@Greenwich.ac.uk (Tim Reis) 
[4] used a Chebyshev pseudospectral method to analyse the dipole-wall collision where the initial trajectory of the dipole is set perpendicularly to the wall then rotate at an angle of $30^{\circ}$ towards one of the no slip walls in a square box. The authors investigated the dissipation of the energy and how it is related to the growth of the enstrophy in a bounded domain. Clercx and Bruneau [5] gave detailed results of two numerical methods, a finite difference method and a pseudospectral Chebyshev method, to simulate normal and oblique dipole-wall collision in a box. The authors presented authoritative data that can be used as benchmark numerical results. They presented the minimum grid resolutions required by their method to simulate this type of flow accurately. However, presumably due to computational limitations, the maximum Reynolds number achieved in [5] is 5000. Later, Wells et al. [6] carried out laboratory and numerical investigations where small scale vorticity in quasi two dimensional square domain was created within the lateral no slip walls. They explained how the collision between the vortex and the wall created a secondary vortex from boundary layer decay. Kramer [7] in his thesis describes the mechanism of the trajectory for normal dipole wall collisions at high Reynolds numbers by using Chebyshev- $\tau$ spectral methods. He gave more details about the small high amplitude vortices that formed from the boundary layer as a result of the collision between the dipole and the boundary. Cieślik et al. [8] examined experimentally the interaction between a dipole in a shallow fluid and a side wall in two dimensional simulations then they made a comparison between results of the experiment and three dimension numerical simulation. Using a finite element method, Guzmán et al. [9] simulated the dipole collision with a no slip sliding wall where the dipole collided with this wall perpendicularly. The sliding wall moves at a constant speed. This type of collision breaks the symmetry of primary vortices while the collision with fixed wall maintains the symmetry between them. Also they explained the role of the Reynolds number on the critical speed in their method. Latt and Chopard [10] used a lattice Boltzmann method with bounce-back boundary conditions and a BGK collision operator to simulate this flow. A reasonable agreement with existing benchmark data was shown. However, they only reported results for a Reynolds number of 625 .

The lattice Boltzmann method (LBM) was originally developed to overcome some of the drawbacks of the lattice gas cellular automaton (LGCA). It has since been derived from a velocity-space truncation and discrtisation of the Boltzmann equation [11, 12, 13, 14, 15, 16]. The Bhatnagar-Gross-Krook collision operator (BGK-LBM) with single relaxation time $\tau$ is the most widely used and popular model $[17,18,19]$. Despite the popularity of the BGK-LBM, it is well-known that its stability suffers at small values of $\tau$ (high Reynolds number flows) [20, 21]. D'Humieres [22] introduced the multiple relaxation time (MRT) model, and such models have been shown to significantly enhance the stability of LBM algorithms with only a little extra computational overhead [23, 20, 24, 25]. A specific and simplified MRT collision operator is the two relaxation time (TRT) model. In TRT models the stability of the algorithm is controlled by just one parameter, the so-called "Magic Parameter" $\Lambda$ which is the product of the relaxation times for even and odd order moments. For the D2Q9 lattice Boltzmann model setting $\Lambda=1 / 4$ is known to offer very good stability properties $[26,27,28]$.

The LBM is distinguished from other methods of computational fluid dynamics (CFD) in several ways. The lattice Boltzmann equation features linear, constant coefficient advection, while traditional numerical methods have 
to discretise the non-linear convection term in the Navier-Stokes equations. Also, the pressure in LBMs come from an equation of state so a Poisson solver is not required. Lattice Boltzmann methods can also incorporate boundary conditions with relative ease. For simple and complex geometries different types of boundary conditions for LBM in many applications have been utilised. The simplest and most common method to apply the no slip boundary condition is "bounce-back". Here, the particles that hit the wall reverse their direction, ready to propagate back to the grid point they came from $[29,15]$. Although often efficient and accurate, bounce-back introduces an additional error into the numerical method; a purely artificial slip. This error term is viscosity-dependent and second order in space if the boundary is placed halfway between grid points, and first order otherwise [16, 30]. Furthermore, Ginzburg and d'Humiereśs [31] presented a general framework for a family of bounce-back methods.

There have been many alternatives and modifications to the most typical LBM boundary condition implementations. Zou and He [32] introduced a "non-equilibrium" bounce back method that eliminates the slip error, but the closure to find the unknowns at the wall is somewhat arbitrary and not immediately extendable to neither larger lattices nor more complicated boundary constraints. Filippova and Hänel [33] consider local grid refinement boundary conditions to deal with curved geometries. Although very useful, this method becomes unstable when the relaxation time is unity. Dupuis and Chopard [34] removed this singularity by taking into account the non equilibrium part of the distribution functions. Bouzidi et al. [35] introduced their interpolation scheme for boundary conditions as a generalisation of the bounce back method with linear or quadratic interpolation for flows in complex geometries. Yu et al. [36] proposed a second order unified scheme to treat the curved solid boundaries which has since been extended by Zhao and Yong [37]. Another popular approach to imposing boundary constraints on the lattice Boltzmann algorithm, especially for particle-laden flows is the immersed boundary method, which guarantees the velocity conditions by adding an artificial force term to the momentum equation - see, for example, [38]. On the other hand, a different methodology has been used to find the unknown distribution functions at a boundary node, one that is based on the hydrodynamic moments of the LBM. Noble et al. [39] used the hydrodynamic moments to apply no-slip boundary conditions for the 6-point FHP lattice. The approach of Noble et al. is not immediately applicable to the now-standard LBM lattices. The FHP model (which is essentially obsolete for simulating fluid flows) has just two unknown functions at a flat boundary while the commonly used D2Q9 lattice has three. Thus a different set of boundary constraints is required. Ginzbourg and d'Humières proposed in [40] their often overlooked local second-order boundary (LSOB) method. This method uses the Chapman-Enskog expansion to second order to find the distributions at a boundary as functions of the LBM moments. Halliday et al. reformulated the LSOB explicitly for the D2Q9 lattice. Lee et al. proposed an implementation of boundary conditions for multi-speed lattices and Chang et al. [41] extended the interpolation method of Ho et al. [42] to curved boundaries. The approach of Chang et al. [41] represents a solid boundary by a set of Lagrangian marks and utilises a second order interpolation, but requires matrix inversions and, like non-equilibrium bounce-back [32], is based on an arbitrary closure. Schlaffer [43] proposed a method to eliminate spurious pressure waves reflecting from the boundaries. Excellent agreement with benchmark results was obtained but additional filtering is needed to achieve the desired goal. There have also been many attempts to impose 

what has been dubbed the "moment-based" method for imposing boundary conditions". The moment-based method is similar in spirit to Noble et al's approach but is far more general in terms of the types of boundary conditions that can be implemented and the lattice stencils they can be applied too. Moment based boundary conditions have been applied to several flows and problems already [46, 21, 46, 44, 47, 48]. However, a detailed study of moment-based boundary conditions for no-slip flows has not been conducted. Comparisons and validation of this new approach are important in assessing its credentials and its potential, including its extension to three dimensions and flows with different boundary conditions.

In this paper we perform a detailed assessment of moment-based boundary conditions for the lattice Boltzmann equation and use them to numerically study the wall-dipole collision flow. We use a TRT model with a new boundary implementation and study the cases when the the dipoles travel towards the wall normally and an angle. A thorough assessment and comparison with benchmark data is performed, and an investigation at Reynolds numbers higher than previously reported is conducted. This sheds light on the physics on the wall-dipole collision flows in these regimes. We also simulate the flows using half-way bounce-back boundary conditions and show that the results obtained with the moment-based method are in closer agreement that bounce-back to results obtained using a spectral method The remainder of the article is organised as follows. In Section 2 we will explain briefly the discrete Boltzmann equation and its use for numerically solving the Navier-Stokes equations. We derive the fully discrete algorithm in Section 3 and the TRT-LBM is discussed in Section 4. The moment-based boundary conditions are introduced in Section 5 and the numerical experiments and discussed in Section 6. Concluding remarks are made in Section 7.

\section{The discrete Boltzmann equation}

In this section we will present briefly the D2Q9 model (see Figure 1) for the discrete velocity Boltzmann equation (DBE), which can be written as

$$
\partial_{t} f_{k}+\boldsymbol{\xi}_{k} \cdot \nabla f_{k}=-\frac{1}{\tau}\left(f_{k}-f_{k}^{(0)}\right), \quad \text { for } k=0, \ldots, 8
$$

The left hand side of equation (1) represents the advection of the particle distribution functions $f_{k}$ with particle velocity $\xi_{k}$. The right hand side is the collision term that relaxes the distribution functions to their local equilibria $f_{k}^{(0)}$ with relaxation time $\tau$.

The equilibrium functions can be obtained from the Maxwell Boltzmann distribution [17] and are given by

$$
f_{k}^{(0)}(\mathbf{x}, t)=\omega_{k} \rho\left(1+\frac{\boldsymbol{\xi}_{k} \cdot \mathbf{u}}{c_{s}^{2}}+\frac{\left(\boldsymbol{\xi}_{k} \cdot \mathbf{u}\right)^{2}}{2 c_{s}^{4}}-\frac{\mathbf{u}^{2}}{2 c_{s}^{2}}\right)
$$

where $\mathbf{u}$ is the macroscopic velocity, $\rho$ is the fluid density, and $c_{s}^{2}=1 / 3$ is the speed of sound for D2Q9 model. The 
weights $\omega_{k}$ have the values

$$
\omega_{k}= \begin{cases}4 / 9, & \text { for } k=0 \\ 1 / 9, & \text { for } k=1, \ldots, 4 \\ 1 / 36, & \text { for } k=5, \ldots, 8\end{cases}
$$

and the discrete lattice velocities $\xi_{k}$ are

$$
\boldsymbol{\xi}_{k}= \begin{cases}(0,0), & k=0 \\ \left(\cos \frac{(k-1) \pi}{2}, \sin \frac{(k-1) \pi}{2}\right), & k=1,2,3,4 \\ \sqrt{2}\left(\cos \frac{(2 k-1) \pi}{4}, \sin \frac{(2 k-1) \pi}{4}\right) . & k=5,6,7,8\end{cases}
$$

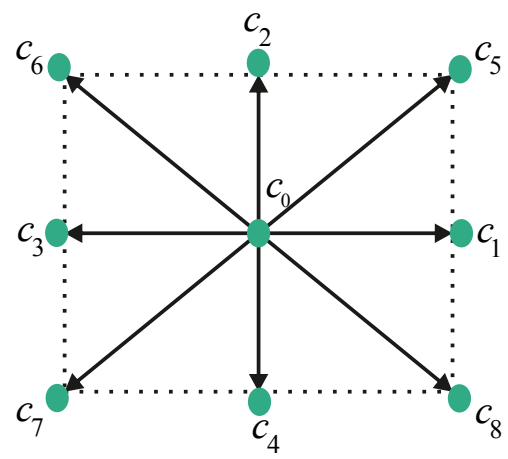

Figure 1: The D2Q9 lattice

The nine discrete velocity moments of the D2Q9 model include hydrodynamic and non-hydrodynamic moments. The 6 hydrodynamic moments are the density $\rho$, momentum $\rho u_{\alpha}$ and momentum flux $\Pi_{\alpha \beta}$ :

$$
\rho=\sum_{k=0}^{8} f_{k} ; \quad \rho u_{\alpha}=\sum_{k=0}^{8} f_{k} \xi_{k \alpha} ; \quad \Pi_{\alpha \beta}=\sum_{k=0}^{8} f_{k} \xi_{k \alpha} \xi_{k \beta}
$$

where $\alpha$ and $\beta$ are the Cartesian components of a vector or tensor. The 3 remaining non-hydrodynamic moments (or the "ghost moments") are

$$
Q_{x x y}=\sum_{k=0}^{8} f_{k} \xi_{k y} \xi_{k x}^{2}, \quad Q_{x y y}=\sum_{k=0}^{8} f_{k} \xi_{k x} \xi_{k y}^{2}, \quad S_{x x y y}=\sum_{k=0}^{8} f_{k} \xi_{k x}^{2} \xi_{k y}^{2}
$$


Taking the first three moment of equation (1) yields the three moment equations

$$
\begin{aligned}
& \partial_{t} \rho+\nabla \cdot \rho \mathbf{u}=0 \\
& \partial_{t} \rho \mathbf{u}+\nabla \cdot \boldsymbol{\Pi}=0 \\
& \partial_{t} \boldsymbol{\Pi}+\nabla \cdot \mathbf{Q}=-\frac{1}{\tau}\left(\boldsymbol{\Pi}-\boldsymbol{\Pi}^{(0)}\right),
\end{aligned}
$$

where the equilibrium momentum flux tensor can be found from equation (2):

$$
\boldsymbol{\Pi}^{(0)}=\frac{\rho}{3} \mathbf{I}+\rho \mathbf{u u}
$$

The moments $\boldsymbol{\Pi}$ and $\mathbf{Q}$ are not conserved in the above system of partial differential equations. Following classical kinetic theory we find the relationship between equation (1) and the Navier-Stokes equations using the ChapmanEnskog expansion. That is, we expand the time derivative and non-conserved moments as follows,

$$
\begin{aligned}
& \partial_{t}=\partial_{t_{0}}+\tau \partial_{t_{1}}+\tau^{2} \partial_{t_{2}}+\ldots \\
& \boldsymbol{\Pi}=\boldsymbol{\Pi}^{(0)}+\tau \mathbf{\Pi}^{(1)}+\tau^{2} \boldsymbol{\Pi}^{(2)}+\ldots \\
& \mathbf{Q}=\mathbf{Q}^{(0)}+\tau \mathbf{Q}^{(1)}+\tau^{2} \mathbf{Q}^{(2)}+\ldots
\end{aligned}
$$

Applying the expansions to equations (7)-(9) and truncating at order $O(\tau)$ allows us to find the first correction to the momentum flux,

$$
\mathbf{\Pi}^{(1)}=-c_{s}^{2} \rho\left(\nabla \mathbf{u}+(\nabla \mathbf{u})^{T}\right)+\mathcal{O}\left(M a^{3}\right)
$$

where $M a=u / c_{s}$ is the Mach number. Thus it can be shown that to first order in $\tau$ the expansion yields the weakly compressible Navier-Stokes equations

$$
\begin{aligned}
\partial_{t} \rho+\nabla \cdot \rho \mathbf{u} & =0 \\
\partial_{t} \rho \mathbf{u}+\nabla \cdot(\rho \mathbf{u u}) & =-\nabla P+\nabla \cdot\left[\nu\left(\nabla \mathbf{u}+\nabla \mathbf{u}^{T}\right)\right]+O\left(M a^{3}\right),
\end{aligned}
$$

where $\nu=\tau c_{s}^{2}$ is the kinematic viscosity and $P=\rho c_{s}^{2}$ is the pressure. 


\section{The lattice Boltzmann equation}

To obtain a fully discrete lattice Boltzmann equation we integrate both sides of equation (1) along a characteristic for time:

$$
f_{k}\left(\mathbf{x}+\boldsymbol{\xi}_{k} \Delta t, t+\Delta t\right)-f_{k}(\mathbf{x}, t)=\int_{0}^{\Delta t} M_{k}\left(\mathbf{x}+\boldsymbol{\xi}_{k} s, t+s\right) d s
$$

where $M_{k}$ is the collision term of DBE. The integration of the left hand side of equation (1) is found exactly while the trapezoidal rule can be used to approximate the right hand side:

$$
f_{k}\left(\mathbf{x}+\xi_{k} \Delta t, t+\Delta t\right)-f_{k}(\mathbf{x}, t)=\frac{\Delta t}{2}\left(M_{k}\left(\mathbf{x}+\boldsymbol{\xi}_{k} \Delta t, t+\Delta t\right)+M_{k}(\mathbf{x}, t)\right)+\mathcal{O}\left(\Delta t^{3}\right)
$$

This is an implicit second order system of equations which is difficult to solve. To obtain an explicit scheme, He et al. [49] defined the transformed functions

$$
\bar{f}_{k}(\mathbf{x}, t)=f_{k}(\mathbf{x}, t)+\frac{\Delta t}{2 \tau}\left(f_{k}(\mathbf{x}, t)-f_{k}^{(0)}(\mathbf{x}, t)\right)
$$

Inserting equation (19) into equation (18) yields the lattice Boltzmann equation in terms of the new variable $\bar{f}_{k}$ :

$$
\bar{f}_{k}\left(\mathbf{x}+\boldsymbol{\xi}_{k} \Delta t, t+\Delta t\right)-\bar{f}_{k}(\mathbf{x}, t)=-\frac{\Delta t}{(\tau+\Delta t / 2)}\left(\bar{f}_{k}(\mathbf{x}, t)-f_{k}^{(0)}(\mathbf{x}, t)\right) .
$$

The conserved moments can be found directly from equation (19)

$$
\begin{aligned}
\rho & =\sum_{k=0}^{8} f_{k}=\sum_{k=0}^{8} \bar{f}_{k}, \\
\rho \mathbf{u} & =\sum_{k=0}^{8} f_{k} \boldsymbol{\xi}_{k}=\sum_{k=0}^{8} \bar{f}_{k} \boldsymbol{\xi}_{k},
\end{aligned}
$$

but the non conserved momentum flux is calculated from

$$
\begin{aligned}
\sum_{k=0}^{8} \bar{f}_{k} \boldsymbol{\xi}_{k} \boldsymbol{\xi}_{k} & =\sum_{k=0}^{8} f_{k}(\mathbf{x}, t) \boldsymbol{\xi}_{k} \boldsymbol{\xi}_{k}+\sum_{k=0}^{8} \frac{\Delta t}{2 \tau} \boldsymbol{\xi}_{k} \boldsymbol{\xi}_{k}\left(f_{k}(\mathbf{x}, t)-f_{k}^{(0)}(\mathbf{x}, t)\right) \\
& =\frac{(2 \tau+\Delta t)}{2 \tau} \boldsymbol{\Pi}-\frac{\Delta t}{2 \tau} \boldsymbol{\Pi}^{(0)}
\end{aligned}
$$

\section{Two relaxation time model}

The two relaxation time (TRT) model is a special case of the of multiple relaxation time (MRT) model [22, 23, $20,50]$. TRT models allow us to enhance the stability of the LBM without the same level of complexity as the full 
MRT collision operator [26, 27, 15]. TRT models can also correct the numerical slip error associated with bounce back boundary conditions but here it is set according to stability requirements alone.

The TRT-DBE relaxes the odd and even order moments at different rates, where odd and even refer to the number of $\boldsymbol{\xi}_{k}$ in the moment. This can be written mathematically as [27]

$$
\partial_{t} f_{k}+\boldsymbol{\xi}_{k} \cdot \nabla f_{k}=-\frac{1}{\tau^{+}}\left(\frac{1}{2}\left(f_{k}+f_{\tilde{k}}\right)-f_{k}^{(0+)}\right)-\frac{1}{\tau^{-}}\left(\frac{1}{2}\left(f_{k}-f_{\tilde{k}}\right)-f_{k}^{(0-)}\right)
$$

where $\tau^{+}$is the relaxation time for even order moments and $\tau^{-}$is the relaxation rate for the odd order moments.

\section{Moment-based boundary conditions}

Moment-based boundary conditions can be considered to be a general methodology for imposing hydrodynamic conditions precisely at grid points in the LBM. After the streaming step of the lattice Boltzmann algorithm, the D2Q9 nodes at planar boundaries aligned with grid points have three incoming distribution functions (pointing into the fluid) that need to be determined. In brief, the moment-based method states that these distribution functions can be found by imposing constraints upon three linearly independent moments at the boundary. To understand the method, let's take a stationary, no-slip, wall at the south of the domain as an example. Here, the three unknown $\bar{f}_{k}$ are $\bar{f}_{2}, \bar{f}_{5}, \bar{f}_{6}$ (see Figure 2). These three unknown functions appear in the moments in one of three different linear combinations, as shown in Table 1. Moments in different rows are linearly independent. Therefore we should impose a constraint (a boundary condition) on one moment from each row in Table 1 , and then solve for the unknown $\bar{f}_{k}$. Since we are interested in numerically solving the Navier-Stokes equations, it seems sensible to choose the hydrodynamic moments instead of the higher order ones that do not appear in these equations of motion. For the no slip condition we will set $u_{x}=u_{y}=0$, and the tangential derivative $\partial_{x} u_{x}=0$. Thus from Table 1 we will pick $\overline{\rho u}_{y}$ from the first row, $\overline{\rho u}_{x}$ 


\begin{tabular}{|c|c|}
\hline Moments & Combination of unknowns at south boundary \\
\hline $\bar{\rho},{\overline{\rho u_{y}}}_{1}, \bar{\Pi}_{y y}$ & $\bar{f}_{2}+\bar{f}_{5}+\bar{f}_{6}$ \\
\hline$\overline{\rho u_{x}}, \bar{\Pi}_{x y}, \bar{Q}_{x y y}$ & $\bar{f}_{5}-\bar{f}_{6}$ \\
\hline $\bar{\Pi}_{x x}, \bar{Q}_{x x y}, \bar{S}_{x x y y}$ & $\bar{f}_{5}+\bar{f}_{6}$ \\
\hline
\end{tabular}

Table 1: Moment combinations for unknown $\bar{f}_{k}$ at the south boundary.

from the second one and for the last equation we will use $\bar{\Pi}_{x x}$ and impose upon them

$$
\overline{\rho u_{x}}=0 ; \quad \overline{\rho u_{y}}=0 ; \quad \bar{\Pi}_{x x}=\rho / 3 .
$$

Since $\Pi_{x x}=\Pi_{x x}^{(0)}+\tau \Pi_{x x}^{(1)}$ (by Chapman-Enskog - see Section 2), the condition on the momentum flux says that $\Pi_{x x}=\Pi_{x x}^{(0)}=\rho / 3$ (since $u_{x}=u_{y}=0$ by the no slip condition). That is, the zero tangential derivative condition is imposed by setting $\Pi_{x x}^{(1)}=0$ (because $\Pi_{x x}^{(1)} \propto \partial_{x} u_{x}$, c.f equation (14)). Solving these equations yields

$$
\begin{aligned}
& \bar{f}_{2}=\bar{f}_{1}+\bar{f}_{3}+\bar{f}_{4}+2\left(\bar{f}_{7}+\bar{f}_{8}\right)-\frac{\rho}{3}, \\
& \bar{f}_{5}=\frac{\rho}{6}-\bar{f}_{1}-\bar{f}_{8}, \\
& \bar{f}_{6}=\frac{\rho}{6}-\bar{f}_{3}-\bar{f}_{7},
\end{aligned}
$$

where the density in the wall is found from the known $\bar{f}_{k}$ and the moment $\overline{\rho u}_{y}$ :

$$
\rho=\overline{\rho u}_{y}+\bar{f}_{0}+\bar{f}_{1}+\bar{f}_{3}+2\left(\bar{f}_{4}+\bar{f}_{7}+\bar{f}_{8}\right) .
$$

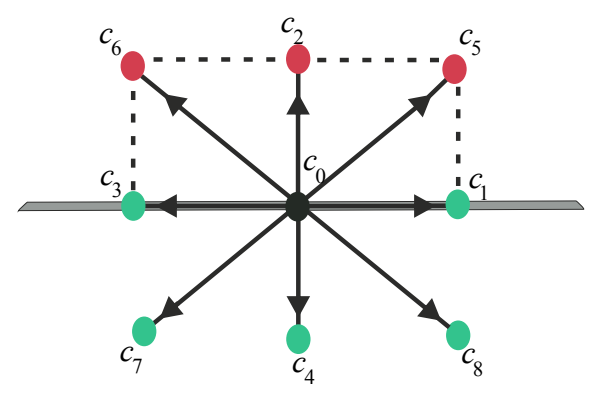

Figure 2: Unknown distribution functions at the south boundary (red colour).

The nodes at the corners need further attention since they have five unknown distribution functions, see Figure 3 . We impose the conditions of the two adjoining walls simultaneously to give the four conditions $\rho u_{x}, \rho u_{y}, \Pi_{x x}$ and $\Pi_{y y}$. The fifth moment is the zero shear stress tensor $\Pi_{x y}$. For example, the five unknown functions at the south east 
boundary be

$$
\begin{aligned}
& \bar{f}_{2}=\frac{2 \rho}{3}-\bar{f}_{0}-\bar{f}_{4}, \\
& \bar{f}_{3}=\frac{2 \rho}{3}-\bar{f}_{0}-\bar{f}_{1}, \\
& \bar{f}_{5}=\frac{\rho}{6}-\bar{f}_{1}-\bar{f}_{8}, \\
& \bar{f}_{6}=-\frac{2 \rho}{3}+\bar{f}_{0}+\bar{f}_{1}+\bar{f}_{4}+\bar{f}_{8}, \\
& \bar{f}_{7}=\frac{\rho}{6}-\bar{f}_{4}-\bar{f}_{8} .
\end{aligned}
$$

Again, the density at this corner is calculated from the above known functions as

$$
\rho=\bar{f}_{0}+2 \bar{f}_{1}+2 \bar{f}_{4}+4 \bar{f}_{8} .
$$

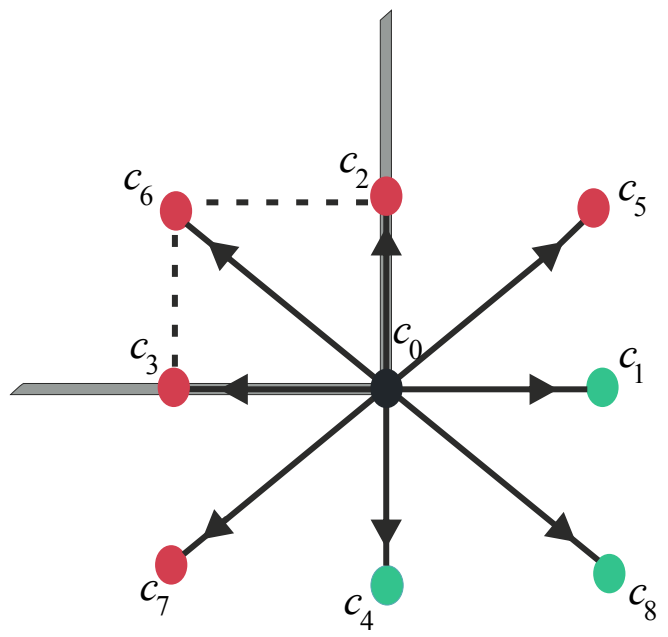

Figure 3: Unknown distribution functions at the south east boundary (red colour).

\section{Dipole-wall collision}

The flow under study is two counter-rotating vortices confined to a square box of size $[-1,1] \times[-1,1]$ with no slip boundary conditions on the walls. The initial vortex is located in the centre at positions $\left(x_{1}, y_{1}\right)$ and $\left(x_{2}, y_{2}\right)$. The initial velocities are

$$
\begin{gathered}
u_{x 0}=-\frac{1}{2}\left|w_{e}\right|\left(y-y_{1}\right) \exp \left(-\left(r_{1} / r_{0}\right)^{2}\right)+\frac{1}{2}\left|w_{e}\right|\left(y-y_{2}\right) \exp \left(-\left(r_{2} / r_{0}\right)^{2}\right) \\
u_{y 0}=\frac{1}{2}\left|w_{e}\right|\left(x-x_{1}\right) \exp \left(-\left(r_{1} / r_{0}\right)^{2}\right)-\frac{1}{2}\left|w_{e}\right|\left(x-x_{2}\right) \exp \left(-\left(r_{2} / r_{0}\right)^{2}\right)
\end{gathered}
$$




\begin{tabular}{|ccc|}
\hline$R e$ & $N_{l b \text { (normal) }}$ & $N_{l b \text { (oblique })}$ \\
\hline 625 & 513 & 513 \\
1250 & 769 & 769 \\
2500 & 1025 & 1025 \\
5000 & 3073 & 4097 \\
\hline
\end{tabular}

Table 2: The minimum resolution for convergence LBM.

where $r_{i}=\sqrt{\left(x-x_{i}\right)^{2}+\left(y-y_{i}\right)^{2}}, r_{0}=0.1$ is the radius of the monopoles, and $w_{e}$ the the strength of the vorticies.

To test our results the total kinetic energy, $E(t)$, and also the total enstrophy, $\Omega(t)$, are calculated

$$
\begin{aligned}
& E(t)=\frac{1}{2} \int_{-1}^{1} \int_{1}^{-1}\left|\mathbf{u}^{2}\right|(\mathbf{x}, t) d x d y, \\
& \Omega(t)=\frac{1}{2} \int_{-1}^{1} \int_{1}^{-1}\left|\omega^{2}\right|(\mathbf{x}, t) d x d y,
\end{aligned}
$$

where $\omega=\partial_{x} u_{y}-\partial_{y} u_{x}$ is the vorticity.

In accordance with the current literature and to allow for consistent comparisons, the initial energy is specified as $E=2$ which is achieved if the strength of the monopole is fixed to be 299.56. We consider two different problems: one where a dipole collides perpendicular with a solid wall and another where the collision is at an oblique angle. The TRT-LBM is used by fixing the parameter $\Lambda=\tau^{+} \tau^{-}=1 / 4$. In our simulations we used a range of Reynolds numbers $(R e)$ from 625 to 10000 for the normal case and 625 to 7500 for the oblique one. Different grid resolutions $N_{l b}$ were employed to test the convergence of the method by monitoring the energy and enstrophy, and the angular momentum in the oblique case only. We consider the results to be convergent when

$$
\left|E\left(N_{l b}\right)-E\left(N_{l b \max }\right)\right| / E(\text { initial }) \leq 0.5 \%,
$$

where $E\left(N_{l b}\right)$ is the energy evaluated at $t=2, E\left(N_{l b \max }\right)$ is the energy at the maximum resolution and $E($ initial $)$ is the initial value for the kinetic energy.

For both normal and oblique wall collisions, higher Reynolds number simulations require higher resolutions for convergence. So in Table 2 we include the minimum resolution required by our algorithm to obtain converged solutions.

\subsection{Normal dipole wall collision}

In this case the two monopoles were located at positions $\left(x_{1}, y_{1}\right)=(0,0.1)$ and $\left(x_{2}, y_{2}\right)=(0,-0.1)$, from where they propel themselves towards the east wall directly. In this section we include the results of LBM at $R e=625$ to $\operatorname{Re}=5000$ and compare them with the results in [5] and [10]. Figure 4 shows the behaviour of the vorticity when the 
dipole moves towards the no slip boundary at $x=1$. Our data is taken at $R e=2500$ with $N_{l b}=2049$. Most of the vorticity is propelled from the centre of the domain to the right boundary. At $t=0.32$ the dipole interacts with the boundary and rebounds from the wall to induce vorticity layers at the boundary where secondary vortices are created. Later, the negative primary vortex detaches from a tertiary thin sheet boundary layer and pairs with positive secondary vortex to form a new dipole then move towards the same wall to hit it again. The same procedure happens to the positive primary vortex Figure 4(d). The thin sheet boundary layer is formed from the gradient of the velocity between the boundary and its nearby region at $t=0.49$. After $t=0.6$ the second collision happens and as a result the wall creates more and wilder vortices at the boundary. Then a secondary dipole is separated from the primary dipole and moves in the opposite direction, see Figure 4(f) which shows the configuration at $t=1.5$. The two 'horseshoe'shapes that initially surround the primary dipole merge to form a new weak dipole which moves towards the left wall at $x=-1$. This dipole hits the wall at $t=1$ and creates another weaker dipole. At this wall and similar to the east wall a thin sheet boundary layer appeared as a result of the variance of the velocity and created other weak vortices. Note that the key for the vortices plot in this figure is similar for all vortices plots in this paper.

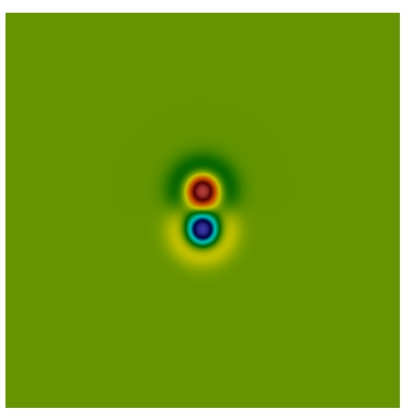

(a) $t=0$

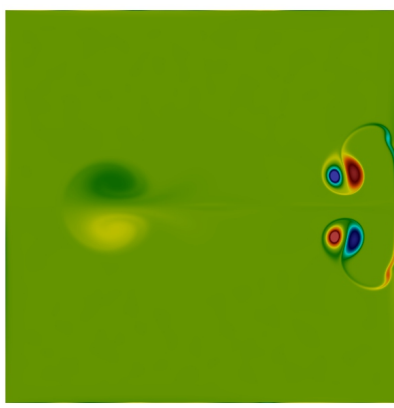

(d) $\mathrm{t}=0.49$

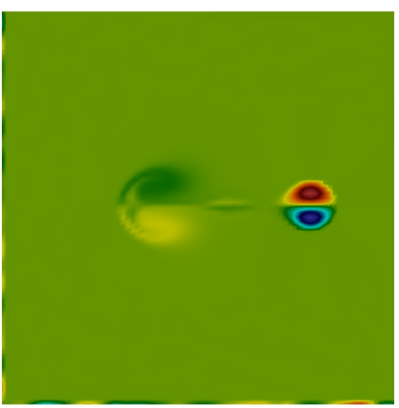

(b) $t=0.2$

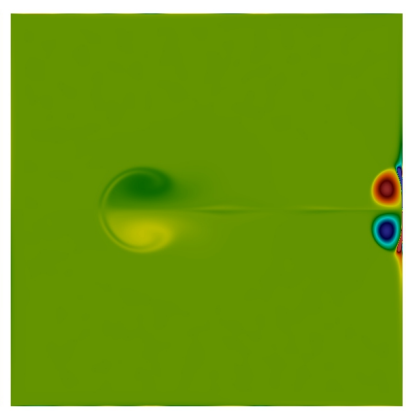

(c) $t=0.32$

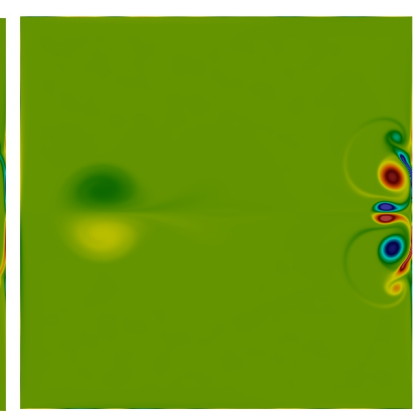

(e) $t=0.617$

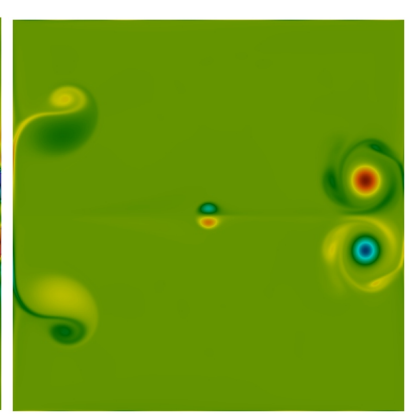

(f) $t=1.5$

Figure 4: Vorticity contours of normal dipole wall collision at $R e=2500$.

In Figure 5 we plot the total kinetic energy $E(t)$ and the total enstrophy $\Omega(t)$ for $R e=2500$ at different grid resolutions, which demonstrates convergence for $N_{L B}=1025$. The kinetic energy begins from the initial value 
$E(0)=2$ then decreases sharply at $t \approx 0.33$. This sharp dissipation in the energy is due to the first dipole collision with the no slip wall which corresponds to an increase in the enstrophy at the same time. At the second dipole-wall collision, $t \approx 0.61$, the dissipation of the energy again increases and synchronizes with the second small peak in the enstrophy.

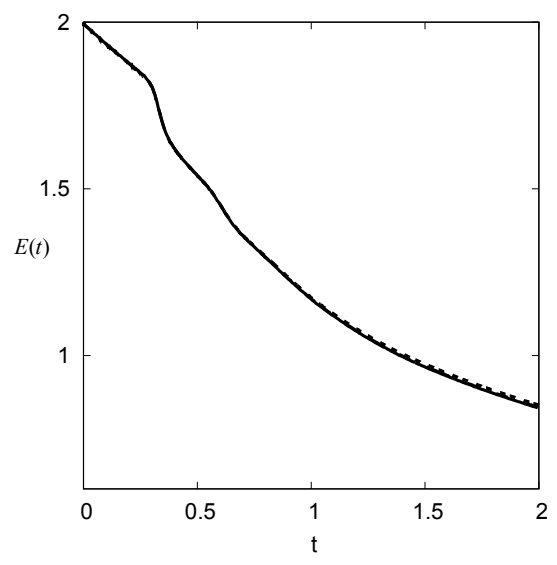

(a) $E(t)$

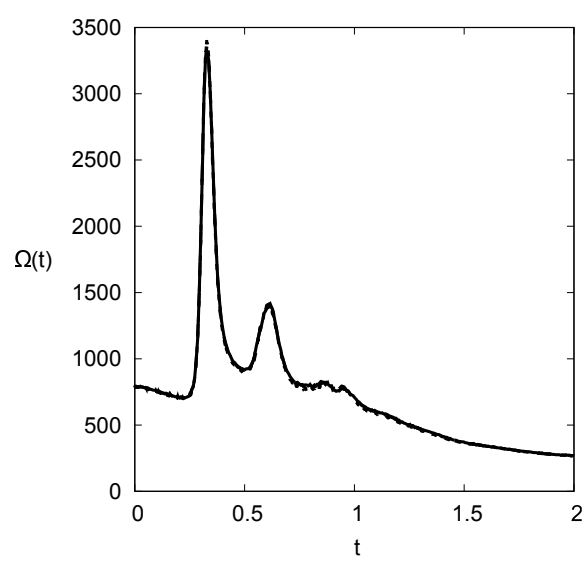

(b) $\Omega(t)$

Figure 5: The kinetic energy and enstrophy for normal dipole wall collision at $R e=2500$ and $N_{l b}=1025$ dotted, 1537 dashed, 2049 line.

In Table 3 the first and second local maxima of the enstrophy $\Omega(t)$ as predicted by the TRT-LBM are shown together with the times they appear (denoted $t_{1}$ and $t_{2}$, respectively). By looking at the results in reference [10] we can see that the value of the first enstrophy peak is close to our result at $R e=625$ where the first enstrophy peak in this reference is $\Omega_{1}=933.8$ at $t=0.371$. Also we compare our results with those obtained from a finite difference method (FDM) and of the pseudospectral Chebyshev method (SM) in reference [5]. Flows at different Reynolds numbers are simulated and we have used the same number of grid points as the finite difference method to give a correct comparison between the present work and the work of [5]. Therefore, the resolutions are used here as follows: $R e=625\left(N_{l b}=1025\right), R e=1250\left(N_{l b}=1537\right), R e=2500\left(N_{l b}=2049\right)$ and $R e=5000\left(N_{l b}=3073\right)$.

\begin{tabular}{|c|c|c|c|c|c|c|c|c|c|c|c|c|}
\hline$R e$ & $t_{1}(\mathrm{LBM})$ & $\Omega_{1}(\mathrm{LBM})$ & $t_{1}(\mathrm{FDM})$ & $\Omega_{1}(\mathrm{FDM})$ & $t_{1}(\mathrm{SM})$ & $\Omega_{1}(\mathrm{SM})$ & $t_{2(\mathrm{LBM})}$ & $\Omega_{2}(\mathrm{LBM})$ & $t_{2}(\mathrm{FDM})$ & $\Omega_{2}(\mathrm{FDM})$ & $t_{2}(\mathrm{SM})$ & $\Omega_{2}(\mathrm{SM})$ \\
\hline 625 & 0.370 & 931.6 & 0.371 & 932.8 & 0.3711 & 933.6 & 0.645 & 306.2 & 0.647 & 305.2 & 0.6479 & 305.2 \\
\hline 1250 & 0.343 & 1884 & 0.341 & 1891 & 0.3414 & 1899 & 0.617 & 727.5 & 0.616 & 724.9 & 0.6162 & 725.3 \\
\hline 2500 & 0.327 & 3305 & 0.328 & 3270 & 0.3279 & 3313 & 0.617 & 1413 & 0.608 & 1408 & 0.6089 & 1418 \\
\hline 5000 & 0.326 & 5496 & 0.323 & 5435 & 0.3234 & 5536 & 0.606 & 3702 & 0.605 & 3667 & 0.6035 & 3733 \\
\hline
\end{tabular}

Table 3: First and second maximum enstrophy $\Omega(t)$ of the dipole by using TRT-LBM. The results are compared with FDM and SM of [5].

The first and second peaks of the enstrophy at the boundary are attributed to a large formulation of the vorticity in the boundary layer. In Figure 6 we plot the vorticity profiles at the boundary $x=1$ and $-0.6 \leq y \leq 0$ at times $t=0.4,0.6,1$ for $R e=625,1250$ and 2500 and we used the same number of resolutions as [5]. The results of the vorticity in our work are in excellent agreement with the results in (Fig. 5) of reference [5]. The vortices at the 
boundary shows the expected behaviour: the vorticity keeps increasing as Reynolds number increases. We can notice that maximum vorticity for $R e=625$ is highest at $t=0.4$, soon after the first dipole/wall collision. At $R e=1250$ and 2500 the the maximum vorticity is highest at $\mathrm{t}=0.6$, around the time of the second collision.

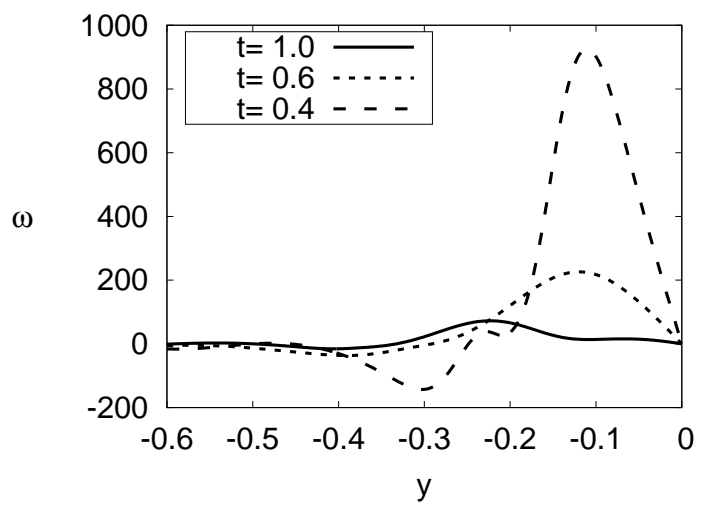

(a) $R e=625$

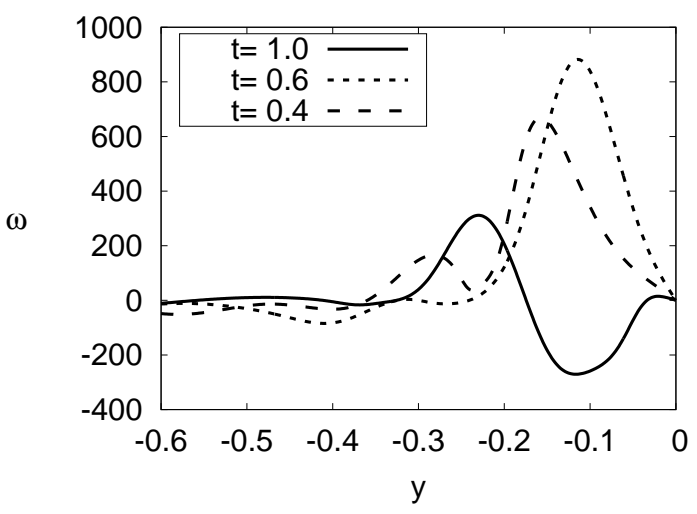

(b) $R e=1250$

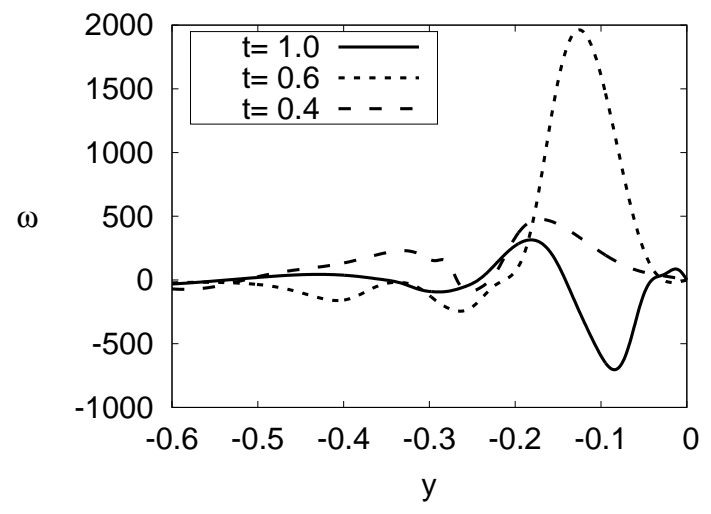

(c) $R e=2500$

Figure 6: The vorticity at the boundary $x=1$ at time $t=1, t=0.4$ and $t=0.6$ at different Reynolds numbers.

To show the effect of the viscosity on the vortices after the dipole collides with the no slip boundary, vorticity profile at $t=1$ are computed. In Figure 7, these vortices are plotted for different Reynolds numbers. We observed the same phenomena as [5]. We can see from these figures that the flow is symmetric for all Reynolds numbers but the behaviour is different in each snapshot. For $R e=2500$ we observe the 'rolling mill' effect as a recently-created dipole is ejected away from the right wall - see Figures 4 (f) and 7(c). At higher Reynolds we get two separate systems that move apart from each other, see Figure 7(d). 


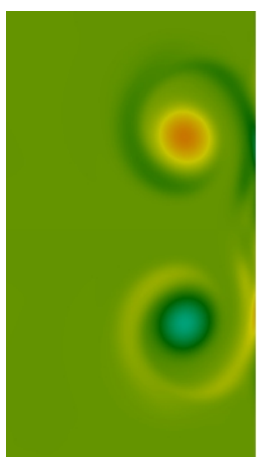

(a) $R e=625$

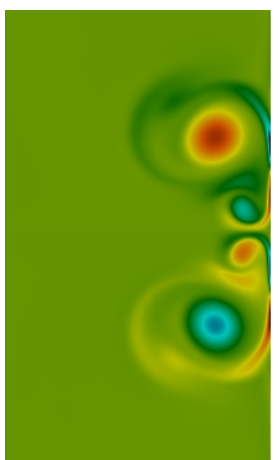

(b) $R e=1250$

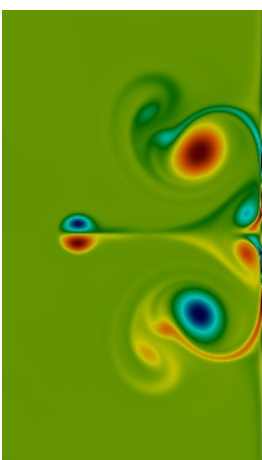

(c) $R e=2500$

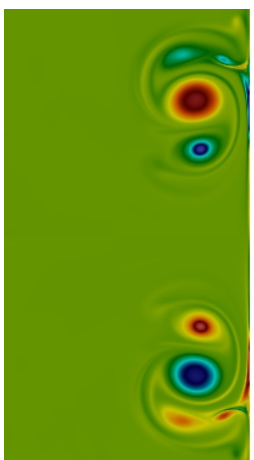

(d) $R e=5000$

Figure 7: Vorticity contours of normal dipole collision wall at $t=1$. vorticity profile shown in the domain $0.3 \leq x \leq 1,-0.6 \leq y \leq 0.6$.

The maximum values of the primary vortex $\omega_{\max }$ and its position $(x, y)$ are inserted in Table 4 . The results are compared with the two methods in [5], the finite difference method and the pseudospectral Chebyshev method. The number of grid points used is the same as those with finite difference method. In our simulations we observed the results of $\omega_{\max }$ at different times $t=0.6,0.625,1$ and $t=1.4$. We can see that the $\omega_{\max }$ increases with Reynolds number. For each Reynolds number the maximum vorticity decreases in time. The maximum vorticity and its location in our simulations are close to the predictions made by other methods. It should be noted that the vorticity is a primary variable in the formulation of the pseudospectral Chebyshev method in [5] while here it is obtained indirectly by numerically differentiating the predicted velocity field. In addition, in Table 5 we present LBM results for the kinetic energy and enstrophy between and after the first and second dipole wall collision and compare them with those in [5]. 


\begin{tabular}{|c|c|c|c|c|c|c|c|}
\hline \multicolumn{4}{|c}{ current work } & \multicolumn{5}{c|}{ Clercx and Bruneau } \\
\hline \multirow{5}{*}{$R e$} & $\mathrm{t}$ & $(x, y)$ & $\omega_{\max }$ & $(x, y)_{(F D)}$ & $(x, y)_{(S M)}$ & $\omega_{\max (F D)}$ & $\omega_{\max (S M)}$ \\
\hline \multirow{5}{*}{625} & 0.6 & $(0.816,0.166)$ & 159.5 & $/$ & $(0.818,0.165)$ & $/$ & 158.9 \\
\cline { 2 - 8 } & 0.625 & $(0.832,0.166)$ & 155.1 & $(0.832,0.166)$ & $/$ & 154.2 & $/$ \\
\cline { 2 - 9 } & 1.0 & $(0.804,0.253)$ & 103.1 & $(0.805,0.254)$ & $(0.805,0.254)$ & 102.6 & 102.6 \\
\cline { 2 - 9 } & 1.4 & $(0.769,0.306)$ & 71.28 & $/$ & $(0.769,0.307)$ & $/$ & 71.0 \\
\hline \multirow{5}{*}{1250} & 0.6 & $(0.872,0.148)$ & 219.6 & $/$ & $(0.874,0.151)$ & $/$ & 219.4 \\
\cline { 2 - 9 } & 0.625 & $(0.884,0.169)$ & 216.5 & $(0.885,0.174)$ & $/$ & 216.1 & $/$ \\
\cline { 2 - 9 } & 1.0 & $(0.847,0.255)$ & 170.9 & $(0.848,0.258)$ & $(0.848,0.257)$ & 170.3 & 170.3 \\
\cline { 2 - 9 } & 1.4 & $(0.808,0.291)$ & 133.2 & $/$ & $(0.809,0.292)$ & $/$ & 132.7 \\
\hline \multirow{5}{*}{2500} & 0.6 & $(0.894,0.161)$ & 260.9 & $/$ & $(0.896,0.165)$ & $/$ & 261.9 \\
\cline { 2 - 8 } & 0.625 & $(0.896,0.193)$ & 259.3 & $(0.896,0.199)$ & $/$ & 260.0 & $/$ \\
\cline { 2 - 8 } & 1.0 & $(0.829,0.219)$ & 231.7 & $(0.826,0.219)$ & $(0.826,0.217)$ & 231.4 & 231.4 \\
\cline { 2 - 8 } & 1.4 & $(0.799,0.192)$ & 202.3 & $/$ & $(0.798,0.195)$ & $/$ & 201.6 \\
\hline \multirow{5}{*}{5000} & 0.6 & $(0.899,0.235)$ & 282.5 & $/$ & $(0.903,0.244)$ & $/$ & 286.9 \\
\hline & 0.625 & $(0.888,0.272)$ & 283.8 & $(0.884,0.275)$ & $/$ & 285.9 & $/$ \\
\cline { 2 - 8 } & 1.0 & $(0.818,0.369)$ & 268.2 & $(0.811,0.367)$ & $(0.811,0.366)$ & 268.6 & 269.1 \\
\hline
\end{tabular}

Table 4: The maximum vorticity $\omega_{\max }$ in a normal wall-dipole collision and its location at $t=0.6,0.625,1,1.4$.

For further investigation and assessment of moment-based conditions we use the TRT LBM with half-way bounceback boundary conditions for comparison. There results are shown in Table 5 and Table 6 . We see that the results computed using bounce-back and moment-based conditions are in good agreement. The data set obtained by using moment-based boundary conditions appears to be more accurate than the data set obtained by using bounce-back in the sense that it is closer agreement with the data set obtained by spectral simulations. This shows that the proposed approach can be a competitive method and gives us confidence to use it to impose physically more complex conditions. 


\begin{tabular}{|c|c|c|c|c|c|c|c|c|c|}
\hline \multicolumn{5}{|c|}{ Lattice Boltzmann method } & \multicolumn{4}{c|}{ Clercx and Bruneau } \\
\hline $\operatorname{Re}$ & $\mathrm{t}$ & $E(t)_{(M M)}$ & $\Omega(t)_{(M M)}$ & $E(t)_{(B B)}$ & $\Omega(t)_{(B B)}$ & $E(t)_{(F D)}$ & $E(t)_{(S M)}$ & $\Omega(t)_{(F D)}$ & $\Omega(t)_{(S M)}$ \\
\hline \multirow{5}{*}{625} & 0.25 & 1.501 & 472.1 & 1.501 & 468.9 & 1.502 & 1.502 & 472.7 & 472.6 \\
\cline { 2 - 10 } & 0.50 & 1.013 & 382.6 & 1.012 & 378.6 & 1.013 & 1.013 & 380.4 & 380.6 \\
\cline { 2 - 10 } & 0.75 & 0.767 & 256.0 & 0.767 & 250.1 & 0.767 & 0.767 & 255.0 & 255.2 \\
\hline \multirow{5}{*}{1250} & 0.25 & 1.719 & 613.6 & 1.719 & 610.0 & 1.721 & 1.720 & 615.0 & 615.0 \\
\cline { 2 - 10 } & 0.50 & 1.312 & 612.8 & 1.311 & 608.1 & 1.313 & 1.313 & 611.3 & 611.9 \\
\cline { 2 - 10 } & 0.75 & 1.061 & 486.2 & 1.061 & 477.8 & 1.061 & 1.061 & 484.4 & 484.7 \\
\hline \multirow{5}{*}{2500} & 0.25 & 1.848 & 725.6 & 1.848 & 718.5 & 1.851 & 1.850 & 727.8 & 728.2 \\
\cline { 2 - 10 } & 0.50 & 1.540 & 917.6 & 1.539 & 909.2 & 1.541 & 1.541 & 916.6 & 920.5 \\
\cline { 2 - 10 } & 0.75 & 1.325 & 809.9 & 1.325 & 794.9 & 1.326 & 1.326 & 805.5 & 808.1 \\
\hline \multirow{3}{*}{5000} & 0.25 & 1.919 & 820.3 & 1.919 & 808.2 & 1.92 & 1.9225 & 822.8 & 823.1 \\
\cline { 2 - 10 } & 0.50 & 1.690 & 1331 & 1.689 & 1317 & 1.692 & 1.692 & 1328 & 1340 \\
\cline { 2 - 9 } & 0.75 & 1.496 & 1539 & 1.495 & 1455 & 1.495 & 1.498 & 1659 & 1517 \\
\hline
\end{tabular}

Table 5: The values of the energy and the enstrophy at different times $0.25,0.50,0.75$ which are before, between and after the first two collisions. Here, MM refers to moment method and BB to bounce back.

\begin{tabular}{|c|c|c|c|c|c|c|c|c|c|c|c|c|}
\hline$R e$ & $t_{1}(\mathrm{BB})$ & $\Omega_{1}(\mathrm{BB})$ & $t_{1}(\mathrm{FDM})$ & $\Omega_{1}(\mathrm{FDM})$ & $t_{1}(\mathrm{SM})$ & $\Omega_{1}(\mathrm{SM})$ & $t_{2}(\mathrm{BB})$ & $\Omega_{2}(\mathrm{BB})$ & $t_{2}(\mathrm{FDM})$ & $\Omega_{2}(\mathrm{FDM})$ & $t_{2}(\mathrm{SM})$ & $\Omega_{2}(\mathrm{SM})$ \\
\hline 625 & 0.376 & 853.7 & 0.371 & 932.8 & 0.3711 & 933.6 & 0.646 & 297.5 & 0.647 & 305.2 & 0.6479 & 305.2 \\
\hline 1250 & 0.344 & 1752 & 0.341 & 1891 & 0.3414 & 1899 & 0.618 & 705.4 & 0.616 & 724.9 & 0.6162 & 725.3 \\
\hline 2500 & 0.327 & 2993 & 0.328 & 3270 & 0.3279 & 3313 & 0.617 & 1352 & 0.608 & 1408 & 0.6089 & 1418 \\
\hline 5000 & 0.327 & 4975 & 0.323 & 5435 & 0.3234 & 5536 & 0.607 & 3394 & 0.605 & 3667 & 0.6035 & 3733 \\
\hline
\end{tabular}

Table 6: First and second maximum enstrophy $\Omega(t)$ of the dipole by using TRT-LBM and BB method. The results are compared with FDM and $\mathrm{SM}$ of [5].

\subsubsection{Higher Reynolds numbers}

In this section we investigate the behaviour of the flow for $R e=7500$ and 10000, (see Figure 8). For these higher Reynolds number the results are converged until $t=0.6$ then begin to diverge significantly. In our simulations, the maximum resolution possible is a grid size of around $4000 \times 4000$. To gauge whether or not this is sufficient, we follow [5] and insist upon having 5 points in the viscous wall boundary layer and estimate the boundary layer thickness as $\delta \approx 1 /(4 \sqrt{R e})$. For $R e=10000$, this gives $N_{l b} \approx 4000$ and here we use $N_{l b}=4097$ and we use this grid size for both $R e=10000$ and $R e=7500$. As for lower Reynolds numbers before $t=0.6$ there are two rapid declines in energy after the first and second wall collisions. Again these correspond with the first and second higher peaks of the enstrophy. 


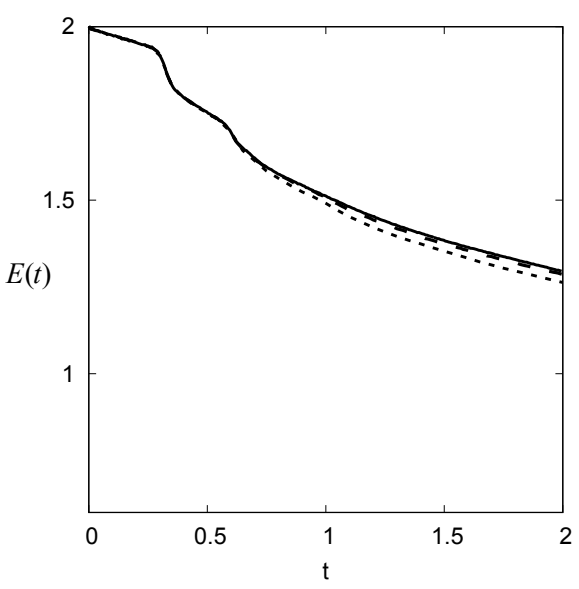

(a) $E(t)$

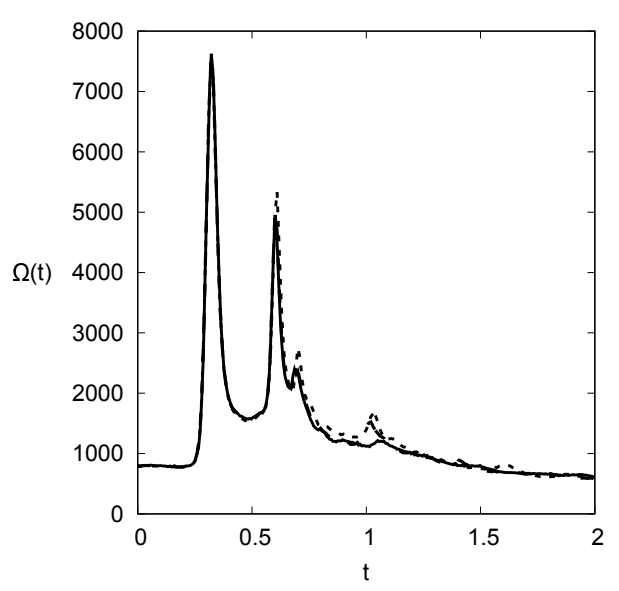

(b) $\Omega(t)$

Figure 8: The kinetic energy and enstrophy for normal dipole wall collision at $R e=7500$ with $N_{l b}=2049$ (dotted), $N_{l b}=3073$ (dashed), $N_{l b}=4097$ (line).

To test the dissipation of the energy at different Reynolds numbers we traced the energy at the time $t=2$ and different Reynolds numbers. We can notice from Table 7 the energy decreases faster with smaller Reynolds number than the larger ones. To make sure this observation is correct we can see the values of $E(t)$ at different time steps in Table 5. The resolutions used are the same as in Tables 4 and 5.

\begin{tabular}{|c|c|c|c|c|c|c|}
\hline$R e$ & 625 & 1250 & 2500 & 5000 & 7500 & 10000 \\
\hline$E(2)$ & 0.305 & 0.518 & 0.841 & 1.108 & 1.285 & 1.359 \\
\hline
\end{tabular}

Table 7: The kinetic energy at $t=2$.

In Figure 9 we made a comparison between high Reynolds number and small Reynolds number, $R e=10000$ and $R e=625$ as an example. We observed that the number of vortices increases with Reynolds number and the affect of the boundary at the rebound of the dipole makes the number of dipoles at $R e=10000$ higher than at $R e=625$. In fact, from Figure 7 (a) and Figure 9 (a) we can notice that the vortices for $R e=625$ decrease in strength as time increases. At $R e=10000$ the space between the upper and lower cores is larger than at the smaller Reynolds number. This behaviour is more obvious at $R e=5000$ and higher, see Figure 7 (d) and Figure 9(b). 


\begin{tabular}{|c||c|c|c|c|c|c|}
\hline$R e$ & $t_{1}$ & $\Omega_{1}(\mathrm{LBM})$ & $t_{2}$ & $\Omega_{2}(\mathrm{LBM})$ & $t_{3}$ & $\Omega_{3}$ (LBM) \\
\hline 7500 & 0.323 & 7626 & 0.604 & 5013 & 0.685 & 2418 \\
\hline 10000 & 0.322 & 9519 & 0.628 & 6455 & 0.721 & 4479 \\
\hline
\end{tabular}

Table 8: First, second and third maxima in enstrophy of the dipole by using TRT-LBM.

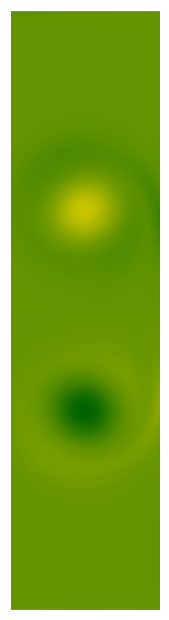

(a) $R e=625$

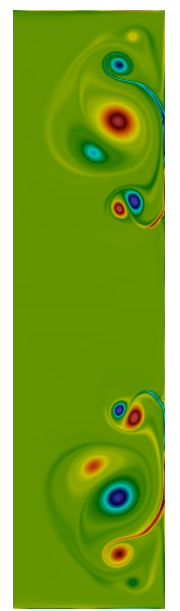

(b) $\quad R e=$ 10000

Figure 9: Snapshot of vorticity at normal dipole collision wall at $t=2$. The domain has been chosen: $0.5 \leq x \leq 1,-1 \leq y \leq 1$.

As a result of the increased activity at higher Reynolds numbers, there are more enstrophy peaks than at smaller Reynolds numbers. This is owing to the increase in the number of wall-vortex collisions. In Table 8 we show the first three maxima in the enstrophy for Reynolds 7500 and 10000. We can notice from Tables 3 and 8 that the ratio between the first and second enstrophy peaks increases with the Reynolds numbers until flattening off after $R e=5000$.

For higher Reynolds numbers, third high strength vortices are observed which are created from the wall boundary layer around $t=0.45$. In Figure 10 the upper dipole is plotted (since it is symmetric with the negative ones). These vortices appear in small circles inside the boundary vortex thin sheet for $R e=2500$ then they become clear at $R e=5000$. For $R e=7500$ these vortices move far from the wall then separate from the sheet at the boundary. For $R e=10000$ a fourth vortex is formed which emerges with the tertiary one, Figure 10 (c). 


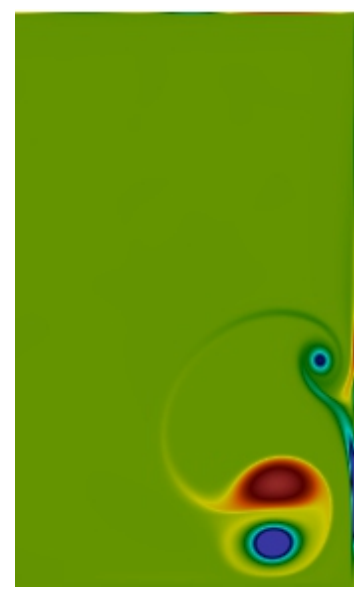

(a) $R e=5000$

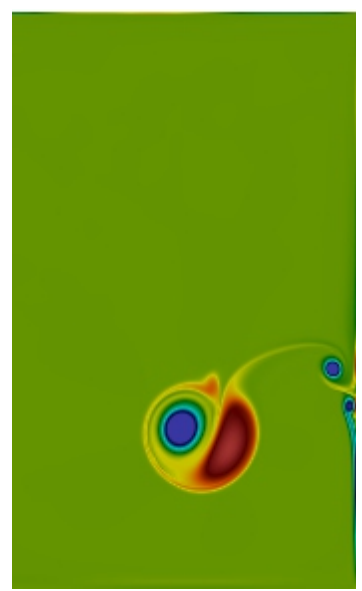

(b) $R e=7500$

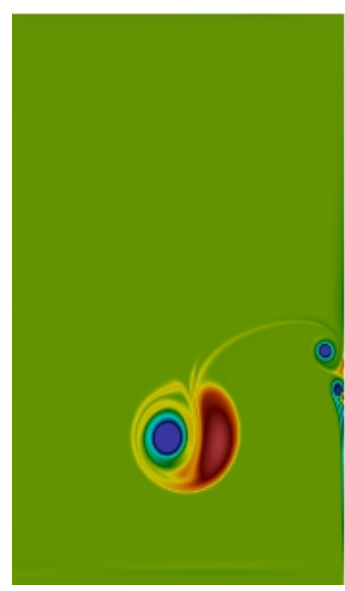

(c) $R e=10000$

Figure 10: Vorticity contours of normal positive dipole collision wall at $t=0.45$. The domain has been chosen: $0.4 \leq x \leq 1,0 \leq y \leq 1$.

We computed the maximum vorticity at different times after the second collision but we found qualitatively different behaviour to the flows at smaller Reynolds numbers. For example Table 9, at $R e=7500$ we noticed that $\omega_{\max }$ at $t=0.6$ is equal to 292.7 then at $t=1$ increased to 497.4 before decreasing again. This is similar to the behaviour of $\omega_{\max }$ at $R e=10000$. This behaviour differs from that of $\omega_{\max }$ in Table 4 . This is due to the formulation of the secondary dipole at the wall after the second collision, as shown in Figure 10. For smaller Reynolds numbers this dipole moves towards the wall then merges with the primary vortices. At higher Reynolds numbers, the secondary dipole moves towards the boundary and rotates after the collision. This then creates tertiary and fourth vortices through interaction with the wall. Figure 11 shows the generated vortices before, (a, b, c), and after, (d, e, f), merging with the primary vortices. The behaviour of creating the strong vortices at the boundary is observed also in [7].

\begin{tabular}{|c|c|c|c|}
\hline$R e$ & $t$ & $(x, y)$ & $\omega_{\max }$ \\
\hline \multirow{4}{*}{7500} & 0.6 & $(0.899,0.265)$ & 293.0 \\
\cline { 2 - 4 } & 0.625 & $(0.888,0.307)$ & 292.6 \\
\cline { 2 - 4 } & 1.0 & $(0.808,0.392)$ & 497.4 \\
\cline { 2 - 4 } & 1.4 & $(0.766,0.488)$ & 341.8 \\
\hline \multirow{4}{*}{10000} & 0.6 & $(0.899,0.231)$ & 297.9 \\
\cline { 2 - 4 } & 0.625 & $(0.899,0.274)$ & 294.5 \\
\cline { 2 - 4 } & 1.0 & $(0.839,0.421)$ & 645.0 \\
\cline { 2 - 4 } & 1.4 & $(0.770,0.522)$ & 401.9 \\
\hline
\end{tabular}

Table 9: The maximum vorticity $\omega_{\max }$ in a normal wall-dipole collision at $R e=7500$ and 10000 , and its location at $t=0.6,0.625,1,1.4$. 


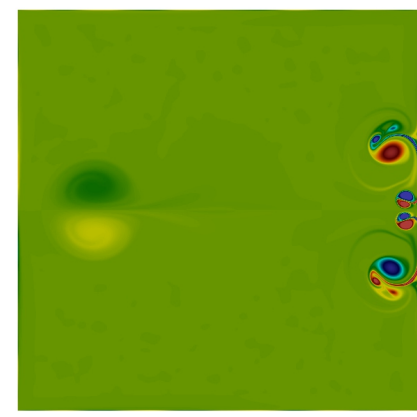

(a) $R e=5000, t=0.55$

240

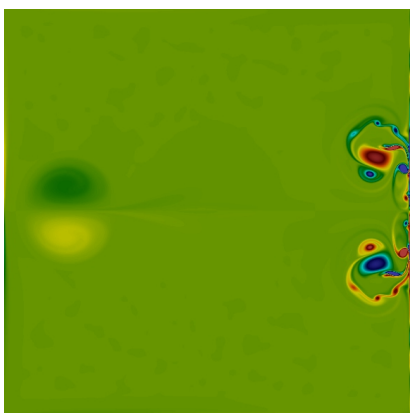

(d) $R e=5000, t=0.63$

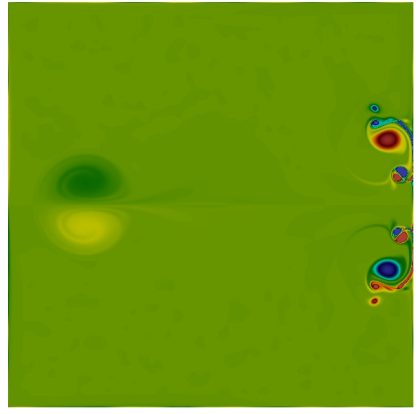

(b) $R e=7500, t=0.64$

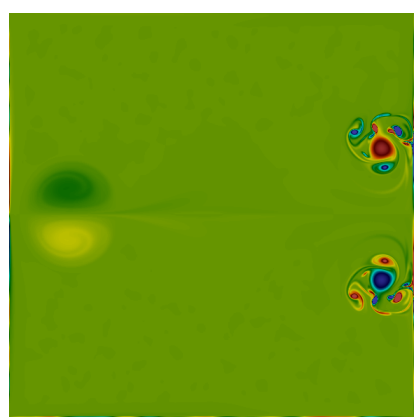

(e) $R e=7500, t=0.74$

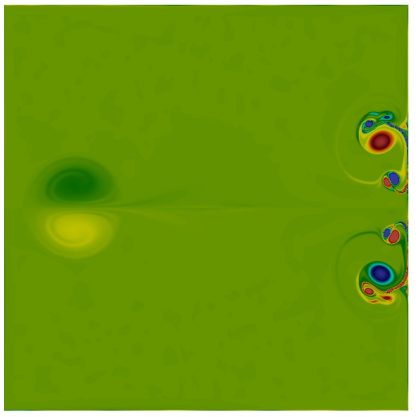

(c) $R e=10000, t=0.66$

Figure 11: Snapshots of the vortices at normal dipole collision wall.

In Figure 12 we calculate the relative error for $R e=625$ with respect to the results with the finest resolution, which in our case is $N_{l b}=3073$. The $L_{2}$-error is computed as $\left|\varphi\left(N_{l b}\right)-\varphi\left(N_{l b \max }\right)\right| / \varphi\left(N_{l b \max }\right)$ where $\varphi$ is the total kinetic energy or total enstrophy. The convergence of this method is shown to be of second order.

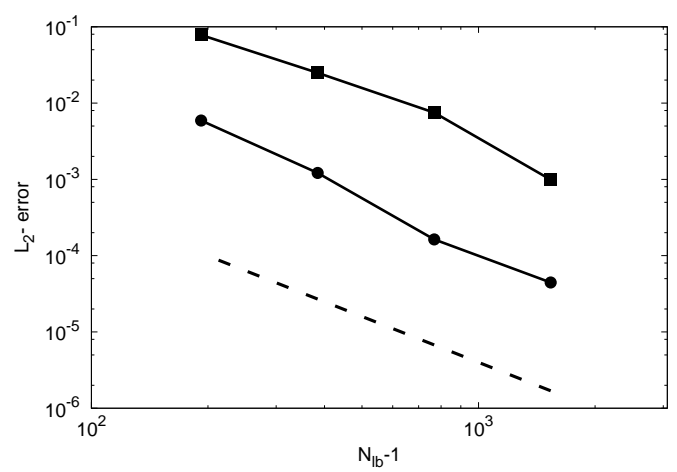

Figure 12: $L_{2}$-error for the enstrophy (fill square) and total kinetic energy (fill circle): normal collision evaluated for $R e=625$ at $t=0.4$. slope 2 is dashed. 


\subsection{Oblique dipole wall collision}

We now consider the case of releasing the dipole at an oblique angle and again compare our results with benchmark data. Here, the dipole rolls from the location $\left(x_{1}, y_{1}\right)=(0.0839,0.0866)$ and $\left(x_{2}, y_{2}\right)=(0.1839,-0.0866)$ towards the no slip wall at $x=1$ at an angle of $30^{\circ}$ to the horizon. Similar to the previous section, we calculate the total enstrophy and total kinetic energy, from equations (33) and (32), at different Reynolds numbers and with various grid resolutions.

Vorticity contours are illustrated in Figure 13 to show the evolution of the dipole at $R e=7500\left(N_{l b}=3073\right)$. From the figures we can see the behaviour of dipole after the second collision, at $t=0.6$, is more vigorous and complex than the normal case. Because of the angle of releasing the dipole towards the wall, the positive and negative monopoles are not symmetric after the first collision with the boundary. Also, as time progresses the primary and secondary vortices move to the upper wall at $y=1$. The two rings that surrounded the dipole at the initial time separate from it interacts with the left corner, which again induces weaker vortices, (see Figure 13 (e)) .

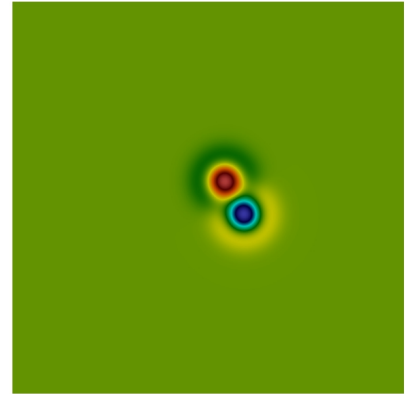

(a) $t=0$

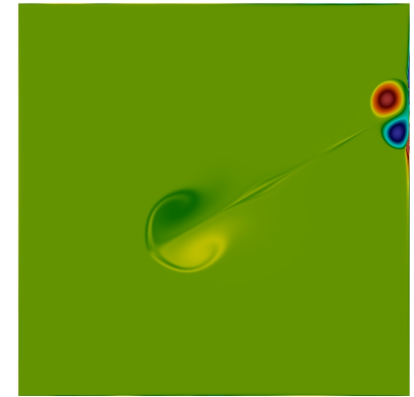

(b) $t=0.3$

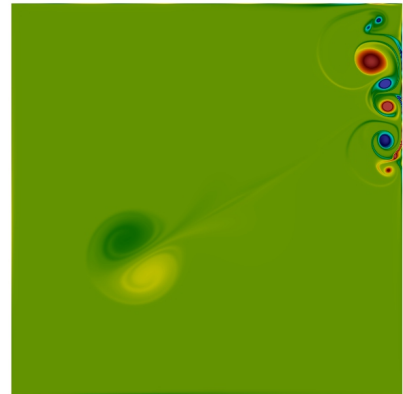

(c) $t=0.6$

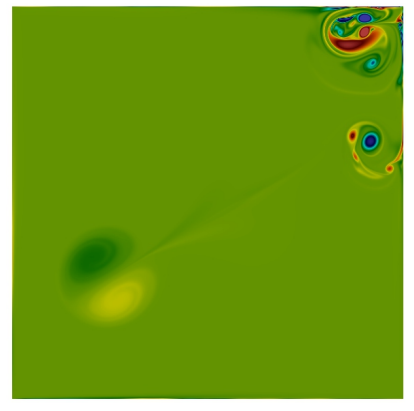

(d) $t=0.8$

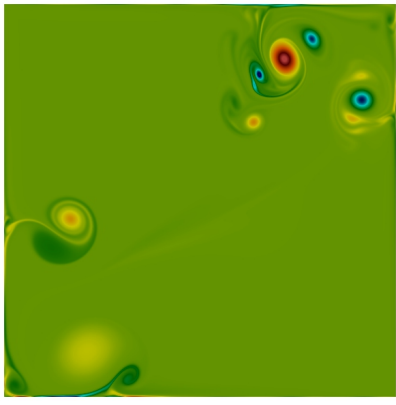

(e) $t=2$

Figure 13: Vorticity contours of oblique dipole collision wall at $\mathrm{Re}=7500$.

Figure 14 plots $E(t)$ and $\Omega(t)$ at $R e=2500$. For different resolutions we observe the convergence of the results at $N_{l b}=1025$. We note that the energy decays rapidly between $0.32<t<0.4$ which is associated with the first peak of the enstrophy. The dissipation of total kinetic energy is less than the dissipation for $R e=2500$ in the normal 
case, see Figure 5. At higher Reynolds numbers, for example 7500, the energy dissipation decreases to less than $R e=2500$. The first peak of the enstrophy is higher than for $R e=2500$ and we can see that the number of peaks increases. Moreover, the additional peaks for high Reynolds numbers less smooth, due to the increase in the number of collisions of additionally created dipoles with the no-slip wall, see Figure 15.

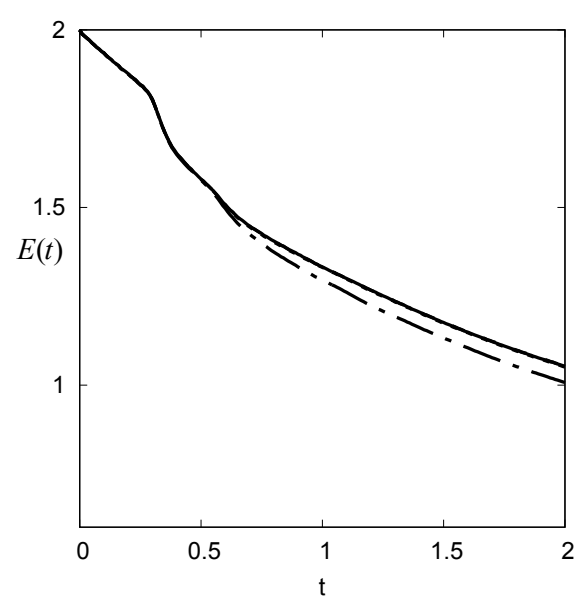

(a) $E(t)$

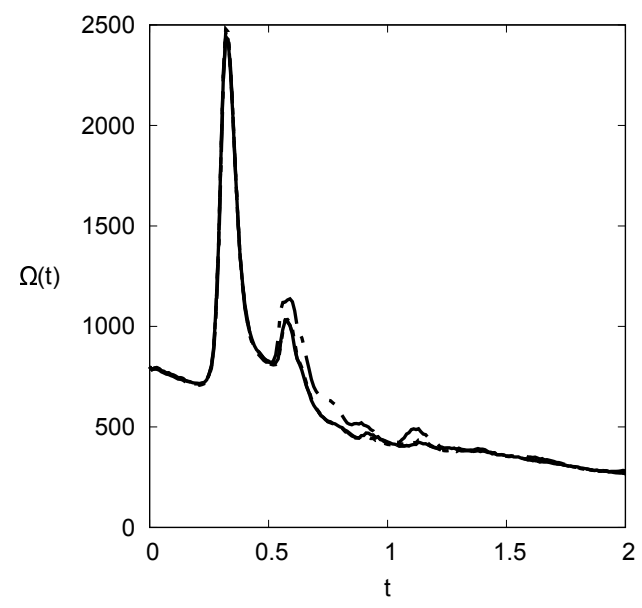

(b) $\Omega(t)$

Figure 14: The kinetic energy and enstrophy for oblique dipole wall collision at $R e=2500$ where $N_{l b}=769$ (dot with dash points), $N_{l b}=1025$ dotted, 1537 dashed and 2049 solid line.

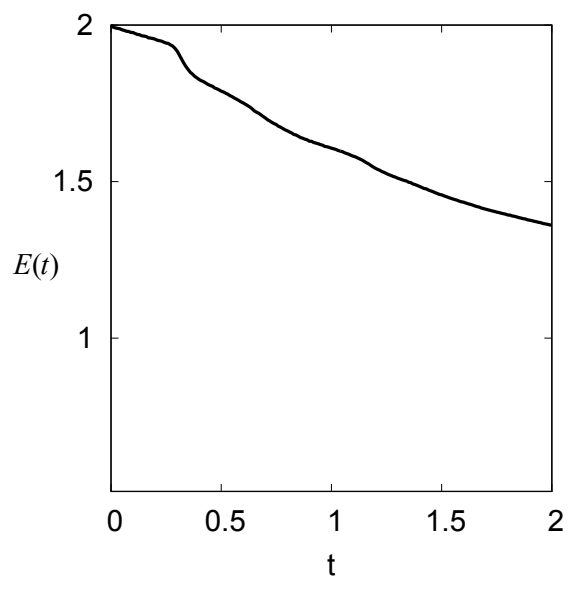

(a) $E(t)$

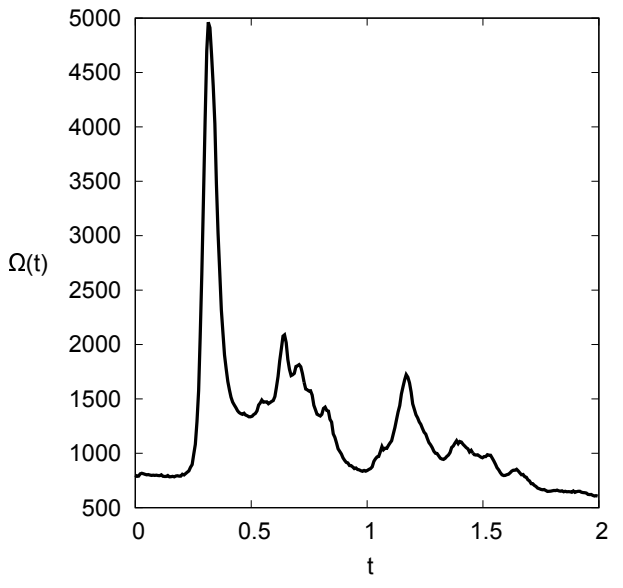

(b) $\Omega(t)$

Figure 15: The kinetic energy and enstrophy for oblique dipole wall collision at $R e=7500, N_{l b}=4097$.

Table 10 shows the computed energy at various times for a range of Reynolds numbers. Clearly the energy consistently decreases less quickly at higher Reynolds number than at lower ones. 


\begin{tabular}{|c|c|c|}
\hline$R e$ & $t$ & $E(t)$ \\
\hline \multirow{3}{*}{625} & 0.3 & 1.423 \\
\cline { 2 - 3 } & 0.5 & 1.049 \\
\cline { 2 - 3 } & 2 & 0.386 \\
\hline \multirow{3}{*}{1250} & 0.3 & 1.659 \\
\cline { 2 - 3 } & 0.5 & 1.353 \\
\cline { 2 - 3 } & 2 & 0.675 \\
\hline \multirow{3}{*}{2500} & 0.3 & 1.790 \\
\cline { 2 - 3 } & 0.5 & 1.579 \\
\cline { 2 - 3 } & 2 & 1.053 \\
\hline \multirow{3}{*}{5000} & 0.3 & 1.886 \\
\cline { 2 - 3 } & 0.5 & 1.729 \\
\cline { 2 - 3 } & 2 & 1.260 \\
\hline \multirow{3}{*}{7500} & 0.3 & 1.916 \\
\cline { 2 - 3 } & 0.5 & 1.789 \\
\cline { 2 - 3 } & 2 & 1.360 \\
\hline
\end{tabular}

Table 10: The kinetic energy at different times and Reynolds numbers

The behaviour of the dipole after colliding with the wall for the oblique case is different from the behaviour of the normal case since the symmetry of the dipole will be broken after the collision, as we will see later. So in this problem we will use another important quantity to test the accuracy of the method which is the total angular momentum. The angular momentum of the flow defined with respect to the centre of the square box is:

$$
L(t)=\int_{-1}^{1} \int_{-1}^{1}\left(x u_{y}(\mathbf{x}, t)-y u_{x}(\mathbf{x}, t)\right) d x d y=-\frac{1}{2} \int_{-1}^{1} \int_{-1}^{1} r^{2} \omega(\mathbf{x}, t) d x d y
$$

In [5], the angular momentum convergence was a sensitive issue. Here, we examined the convergence of the results for angular momentum at different grid resolutions. As a result, we found that the behaviour of the angular momentum matches the results in [5]. For example, Figure 16 shows that the results for $L(t)$ at $R e=625$ have converged for $N_{l b}=769$. At $R e=2500$ convergence is achieved at $N_{l b}=1537$. For $R e=7500$ the results have not converged for $N_{l b}=3073$. Overall, from these figures the angular momentum increases with time also the rate of change of $L(t)$ increases with the Reynolds number. Moreover, the frequently of the oscillations of the results become higher with higher Reynolds numbers 


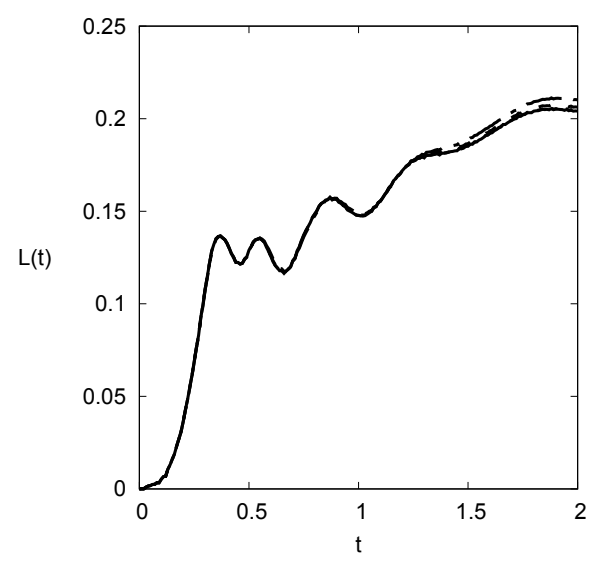

(a) $R e=625: N_{l b}=513$ (dot with dash points), $N_{l b}=769$ (dashed), $N_{l b}=1537$ (dotted) and $N_{l b}=2049$ (line)

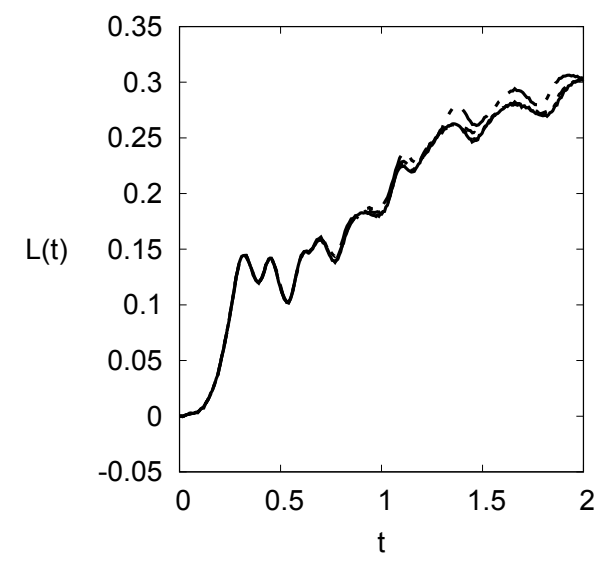

(b) $R e=2500: N_{l b}=1025$ (dot with dash points), $N_{l b}=1537$ (dashed) $N_{l b}=2049$ (dotted) and $N_{l b}=3073$ (line)

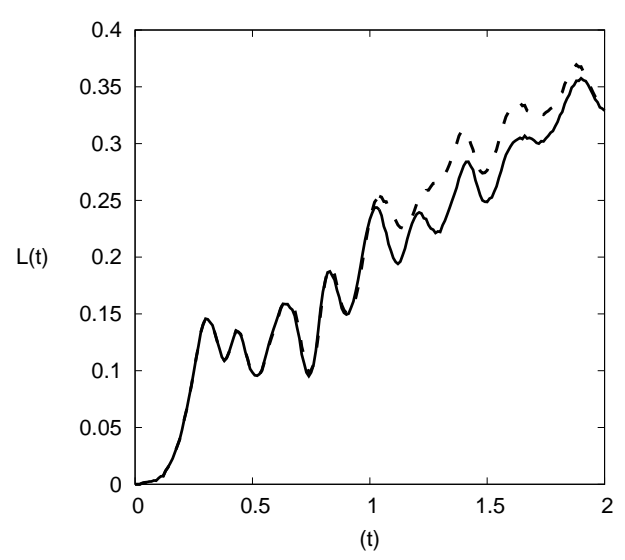

(c) $R e=7500: N_{l b}=3073$ (dashed) and $N_{l b}=4097$ (line)

Figure 16: The total angular momentum for oblique dipole wall collision at different Reynolds numbers.

Table 11 shows $\Omega(t)$ at the first and second dipole collision with the east wall at $x=1$. We compare our results with data obtained from the finite difference method and the pseudospectral Chebyshev method reported in [5] at different Reynolds numbers. Here we have used the same number of grid points as the finite difference method. The results are in very good agreement with those presented in [5]. 


\begin{tabular}{|c|c|c|c|c|c|c|c|c|c|c|c|c|}
\hline$R e$ & $t_{1}(\mathrm{LBM})$ & $\Omega_{1}$ (LBM) & $t_{1(\mathrm{FDM})}$ & $\Omega_{1}(\mathrm{FDM})$ & $t_{1}(\mathrm{SM})$ & $\Omega_{1}(\mathrm{SM})$ & $t_{2}(\mathrm{LBM})$ & $\Omega_{2}(\mathrm{LBM})$ & $t_{2}(\mathrm{FDM})$ & $\Omega_{2}(\mathrm{FDM})$ & $t_{2}(\mathrm{SM})$ & $\Omega_{2}(\mathrm{SM})$ \\
\hline 625 & 0.362 & 778.3 & 0.360 & 766.6 & 0.359 & 768.0 & 0.645 & 306.2 & 0.6435 & 304.5 & 0.6435 & 304.5 \\
\hline 1250 & 0.333 & 1485 & 0.335 & 1473 & 0.335 & 1478 & 0.582 & 692.9 & 0.581 & 689.4 & 0.5819 & 688.8 \\
\hline 2500 & 0.324 & 2455 & 0.323 & 2435 & 0.323 & 2447 & 0.569 & 1029 & 0.569 & 1024 & 0.5692 & 1024 \\
\hline 5000 & 0.318 & 3813 & 0.317 & 3769 & 0.317 & 3825 & 0.591 & 1679 & 0.591 & 1707 & 0.5936 & 1683 \\
\hline 7500 & 0.320 & 4966 & $/$ & $/$ & $/$ & $/$ & 0.65 & 2008 & $/$ & $/$ & $/$ & $/$ \\
\hline
\end{tabular}

Table 11: First and second maximum enstrophy of Oblique wall dipole collision using moment method with TRT-LBM. The results are compared with FDM and SM of [5].

Similar to the normal case we applied the half-way bounce-back with TRT-LBM for the purpose of comparison with moment-based conditions. These results are given in Table 12 where we once again see that moment-based approach predicts results in better agreement with spectral simulations than bounce-back.

\begin{tabular}{|c|c|c|c|c|c|c|c|c|c|c|c|c|}
\hline$R e$ & $t_{1(\mathrm{BB})}$ & $\Omega_{1}(\mathrm{BB})$ & $t_{1}(\mathrm{FDM})$ & $\Omega_{1}(\mathrm{FDM})$ & $t_{1}(\mathrm{SM})$ & $\Omega_{1}(\mathrm{SM})$ & $t_{2}(\mathrm{BB})$ & $\Omega_{2}(\mathrm{BB})$ & $t_{2}(\mathrm{FDM})$ & $\Omega_{2}(\mathrm{FDM})$ & $t_{2}(\mathrm{SM})$ & $\Omega_{2}(\mathrm{SM})$ \\
\hline 625 & 0.364 & 722.8 & 0.360 & 766.6 & 0.395 & 768.0 & 0.647 & 295.6 & 0.6435 & 304.5 & 0.6435 & 304.5 \\
\hline 1250 & 0.333 & 1385 & 0.335 & 1473 & 0.335 & 1478 & 0.583 & 664.5 & 0.581 & 689.4 & 0.5819 & 688.8 \\
\hline 2500 & 0.325 & 2253 & 0.323 & 2435 & 0.323 & 2447 & 0.570 & 979.5 & 0.569 & 1024 & 0.5692 & 1024 \\
\hline 5000 & 0.319 & 3567 & 0.317 & 3769 & 0.317 & 3825 & 0.591 & 1602 & 0.591 & 1707 & 0.5936 & 1683 \\
\hline
\end{tabular}

Table 12: First and second maximum enstrophy of Oblique wall dipole collision using bounce-back with TRT-LBM. The results are compared with FDM and SM of [5].

The vorticity $\omega$ at the boundary $x=1$ and $-0.5 \leq y \leq 1$ is plotted in Figure 17. In this figure we show the behaviour of the vorticity at different times, including after the first and second collision with the right wall. The data obtained at $R e=625,1250,2500$ and 7500 at time $t=0.4,0.6$ and 1 . To make a comparison between our results and [5] we used the same resolutions as finite difference method in that reference. From the figure we note, as from [5], that the behaviour of the vorticity at the boundary in the oblique dipole wall collision is more complex than the normal collision case. Similar to the normal case, at $R e=625$ the maximum vorticity at the wall is highest at $\mathrm{t}=0.4$ whilst at higher Reynolds numbers it is greatest at $t=0.6$. Also, in each case the highest maximum vorticity is roughly equal in magnitude to the lowest minimum vorticity, as would be the case for the normal collision. Furthermore, additional local maxima appear at later times for higher $R e$, probably associated with more complex flow patterns due to enhanced vortex creation . 


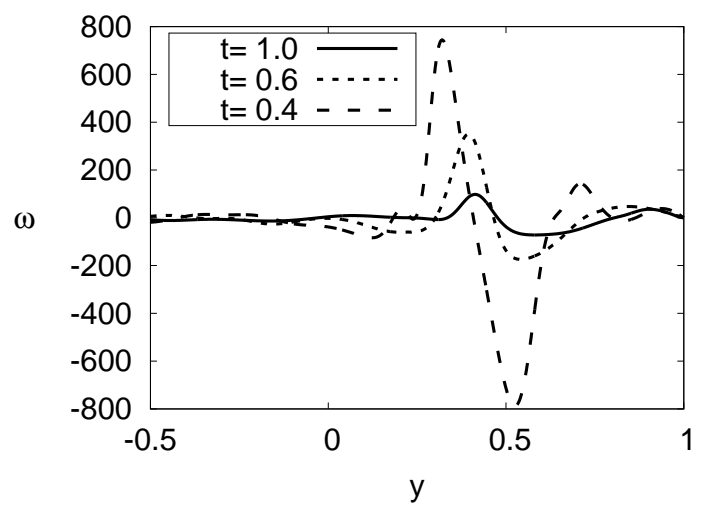

(a) $\operatorname{Re}=625$

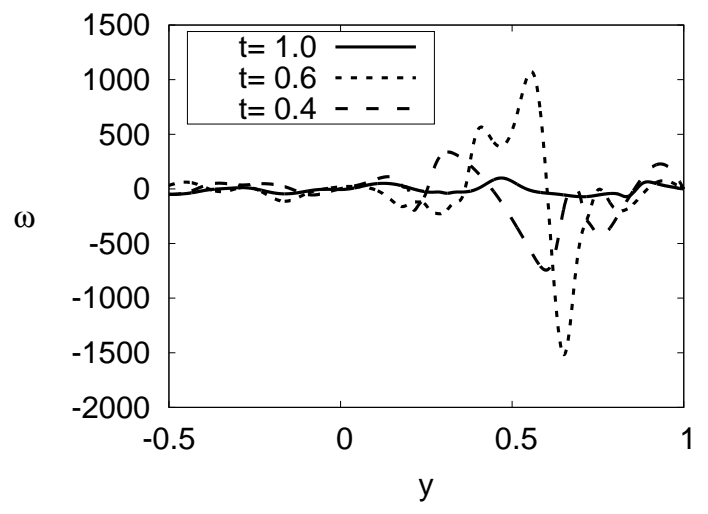

(c) $R e=2500$

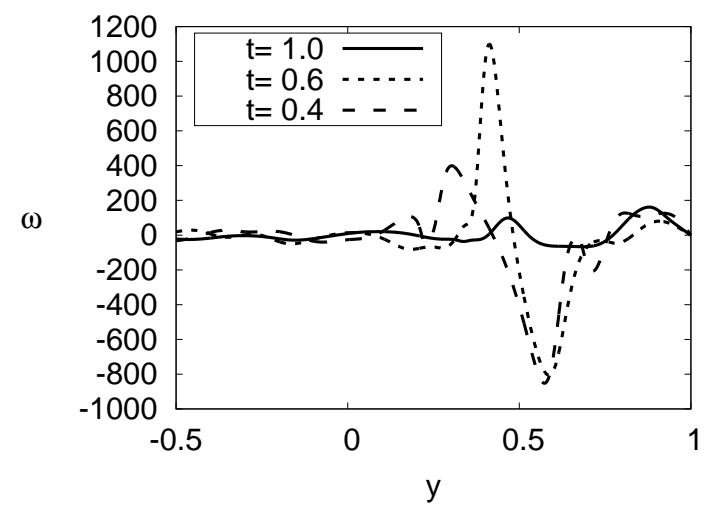

(b) $R e=1250$

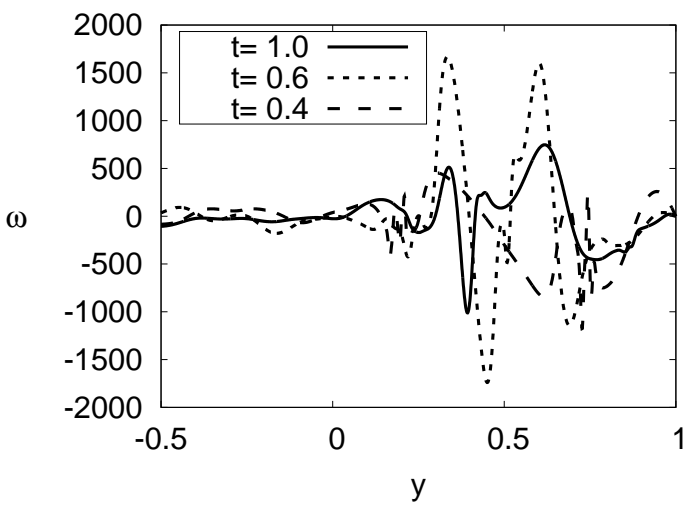

(d) $R e=7500$

Figure 17: The vorticity at the boundary $x=1$ at time $t=1, t=0.4$ and $t=0.6$ at different Reynolds number: (a) $N_{l b}=1537$, (b) $N_{l b}=2049$, (c) $N_{l b}=3073$, (d) $N_{l b}=3073$.

After $t=0.45$ small third vortices start to form from the thin filament sheet that is induced at the boundary for $R e=5000$ and 7500. Then as time progresses a fourth vortex appears at the positive monopole, Figure 18(a, b, c). After $t=0.6$ the top two vortices banded with others near the top corner and merge with the primary vortex, see Figure 18(d, e, f). At smaller Reynolds numbers these vortices are not present at the positive side of the sheet but we can see them clearly on the negative side. We should mention that these small vortices have the same higher amplitude as the primary dipole. 


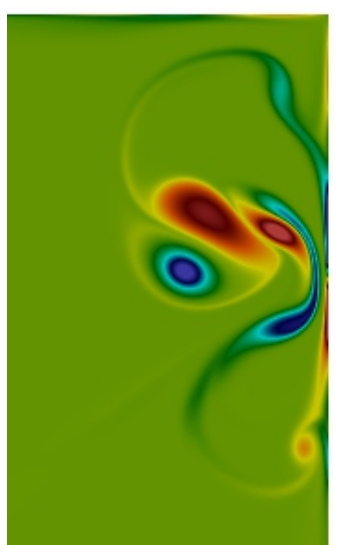

(a) $R e=2500$

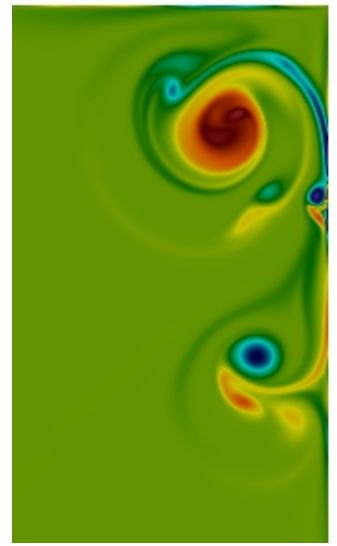

(d) $R e=2500$

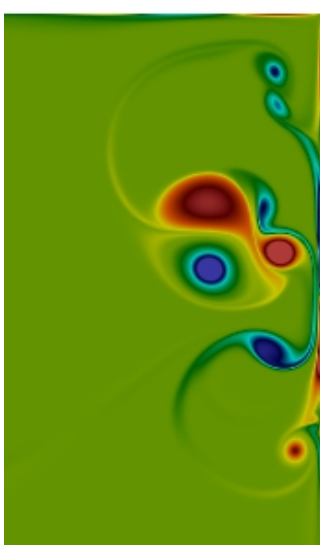

(b) $R e=5000$

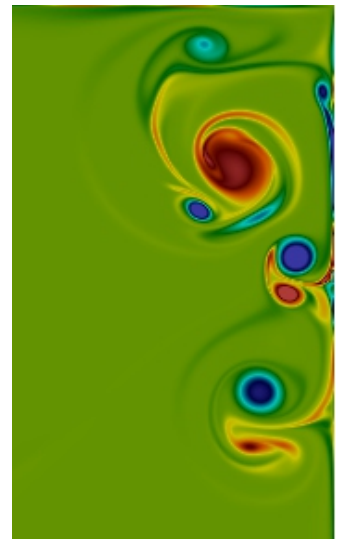

(e) $R e=5000$

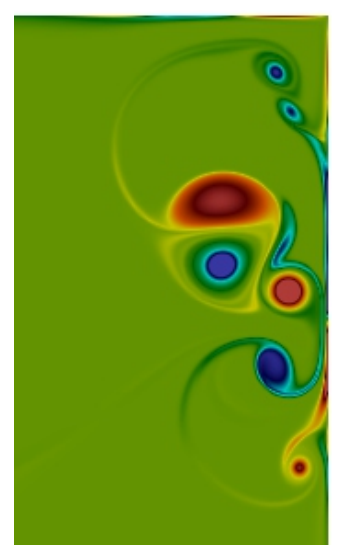

(c) $R e=7500$

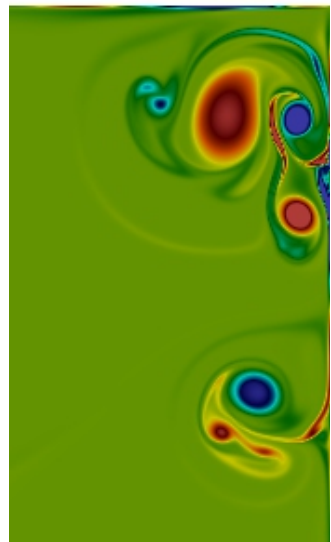

(f) $R e=7500$

Figure 18: Vorticity snapshots of oblique dipole collision wall at $t=0.55$ and $t=0.7$ inserted in the first then second row respectively. The domain has been shown: $0.4 \leq x \leq 1,0 \leq y \leq 1$.

To better understand the behaviour of the dipole at the boundary after the second collision, in Figure 19 we plot vorticity contours for different Reynolds numbers. In this case the most interesting behaviour of the vorticity is at the top right corner where the dipoles interact with each other after the second collision. In these figures the computations are shown in the domain $0.3 \leq x \leq 1$ and $0 \leq y \leq 1$ at $t=1$. Here, as the Reynolds number is increased, the vortex collisions near the corner becomes more energetic creating further vortices. Additional vortices are also created by interactions with the top wall, leading to extremely complex flow patterns. 


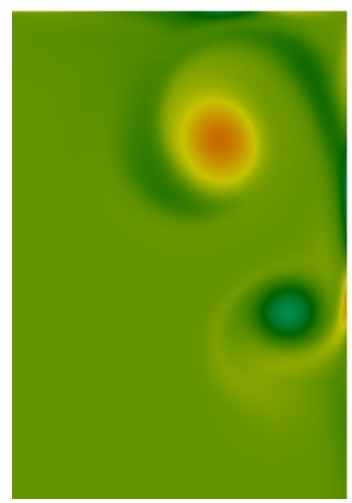

(a) $R e=625$

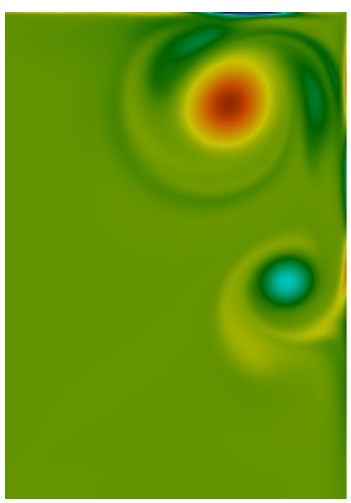

(b) $R e=1250$

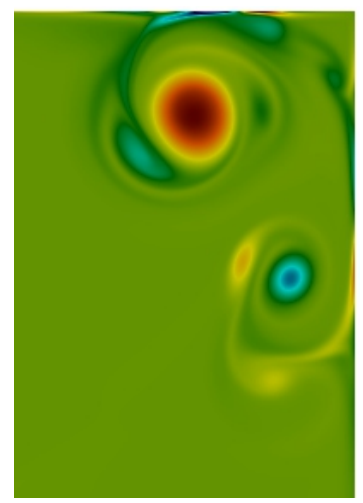

(c) $R e=2500$

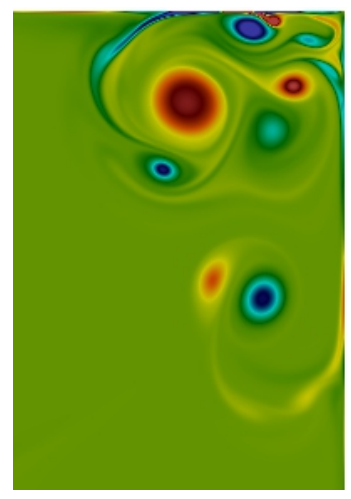

(d) $R e=5000$

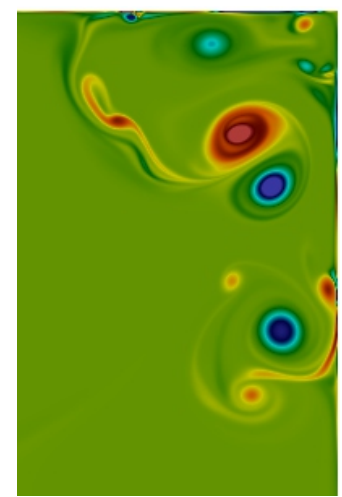

(e) $R e=7500$

Figure 19: Vorticity contours of oblique dipole collision wall at $t=1$. The vorticity are chosen in the domain $0.3 \leq x \leq 1$ and $0 \leq y \leq 1$.

In Tables 13 and 14 we pick out the maximum and minimum vorticity and the location of these extrema. These data shown for various Reynolds numbers at different times. We can see in general that the strength of the maximum vortices at each Reynolds number gradually decrease between $t=0.6$ and $t=1.8$. However, for $R e=7500$ small but intense vortices are formed near the top right corner as the dipole interacts with the corner. These small vortices have a significant impact on the results, causing fluctuations in the maximum vorticity until $t=1.4$. 


\begin{tabular}{|c|c|c|c|c|c|c|c|}
\hline \multicolumn{4}{|c|}{ current work } & \multicolumn{4}{|c|}{ Clercx and Bruneau } \\
\hline$R e$ & $\mathrm{t}$ & $(x, y)$ & $\omega_{\max }$ & $(x, y)_{(F D)}$ & $(x, y)_{(S M)}$ & $\omega_{\max (F D)}$ & $\omega_{\max (S M)}$ \\
\hline \multirow{3}{*}{625} & 0.6 & $(0.740,0.658)$ & 161.9 & $(0.740,0.659)$ & $(0.740,0.659)$ & 161.4 & 161.5 \\
\hline & 1.2 & $(0.713,0.770)$ & 94.09 & $(0.711,0.771)$ & $(0.712,0.772)$ & 93.74 & 93.80 \\
\hline & 1.8 & $(0.592,0.717)$ & 63.06 & $(0.591,0.716)$ & $(0.592,0.717)$ & 63.0 & 63.0 \\
\hline \multirow{3}{*}{1250} & 0.6 & $(0.800,0.606)$ & 220.8 & $(0.807,0.607)$ & $(0.807,0.607)$ & 220 & 220 \\
\hline & 1.2 & $(0.691,0.777)$ & 157.5 & $(0.691,0.779)$ & $(0.691,0.779)$ & 157.0 & 157.0 \\
\hline & 1.8 & $(0.553,0.754)$ & 119.9 & $(0.551,0.754)$ & $(0.550,0.753)$ & 119.6 & 119.7 \\
\hline \multirow{3}{*}{2500} & 0.6 & $(0.813,0.625)$ & 344. & $(0.900,0.690)$ & $(0.899,0.689)$ & 258.7 & 258.7 \\
\hline & 1.2 & $(0.613,0.788)$ & 216.7 & $(0.609,0.789)$ & $(0.608,0.788)$ & 216.6 & 217.0 \\
\hline & 1.8 & $(0.578,0.685)$ & 192.1 & $(0.555,0.694)$ & $(0.560,0.691)$ & 191.2 & 191.6 \\
\hline \multirow{3}{*}{5000} & 0.6 & $(0.789,0.666)$ & 285.4 & $(0.794,0.680)$ & $(0.797,0.684)$ & 288 & 288 \\
\hline & 1.2 & $(0.570,0.732)$ & 261.4 & $(0.643,0.710)$ & $(0.612,0.712)$ & 264 & 264 \\
\hline & 1.8 & $(0.618,0.743)$ & 240.6 & I & I & I & I \\
\hline \multirow{3}{*}{7500} & 0.6 & $(0.840,0.704)$ & 293.1 & I & I & I & I \\
\hline & 1.2 & $(0.695,0.856)$ & 493.7 & I & I & I & I \\
\hline & 1.8 & $(0.496,0.757)$ & 359.6 & I & I & I & I \\
\hline
\end{tabular}

Table 13: The maximum vorticity $\omega_{\max }$ at positive vortex in a oblique wall-dipole collision and its location at $t=0.6,1.2,1.8$. 


\begin{tabular}{|c|c|c|c|c|c|c|c|}
\hline \multicolumn{4}{|c|}{ current work } & \multicolumn{4}{|c|}{ Clercx and Bruneau } \\
\hline$R e$ & $\mathrm{t}$ & $(x, y)$ & $\omega_{\min }$ & $(x, y)_{(F D)}$ & $(x, y)_{(S M)}$ & $\omega_{\min (F D)}$ & $\omega_{\min (S M)}$ \\
\hline \multirow{3}{*}{625} & 0.6 & $(0.897,0.361)$ & -152.9 & $(0.898,0.365)$ & $(0.898,0.364)$ & -151.8 & -151.9 \\
\hline & 1.2 & $(0.871,0.434)$ & -60.33 & $(0.872,0.435)$ & $(0.872,0.436)$ & -59.9 & -59.9 \\
\hline & 1.8 & $(0.886,0.680)$ & -29.37 & $(0.888,0.685)$ & $(0.887,0.685)$ & -29.2 & -29.1 \\
\hline \multirow{3}{*}{1250} & 0.6 & $(0.928,0.383)$ & -213.4 & $(0.928,0.381)$ & $(0.928,0.381)$ & -212.5 & -212.7 \\
\hline & 1.2 & $(0.877,0.513)$ & -108.6 & $(0.877,0.514)$ & $(0.877,0.513)$ & -108.3 & -108.3 \\
\hline & 1.8 & $(0.811,0.872)$ & -63.15 & $(0.811,0.867)$ & $(0.812,0.865)$ & -63.16 & -63.16 \\
\hline \multirow{3}{*}{2500} & 0.6 & $(0.912,0.553)$ & -341.4 & $(0.923,0.368)$ & $(0.923,0.368)$ & -248.2 & -248.3 \\
\hline & 1.2 & $(0.906,0.488)$ & -154.3 & $(0.906,0.484)$ & $(0.907,0.492)$ & -155.1 & -154.9 \\
\hline & 1.8 & $(0.844,0.763)$ & -102.9 & $(0.841,0.746)$ & $(0.841,0.738)$ & -104.0 & -103.7 \\
\hline \multirow{3}{*}{5000} & 0.6 & $(0.893,0.320)$ & -277.9 & $(0.892,0.320)$ & $(0.894,0.319)$ & -278 & -278 \\
\hline & 1.2 & $(0.527,0.599)$ & -340.0 & $(0.909,0.470)$ & I & -224 & I \\
\hline & 1.8 & $(0.733,0.812)$ & -204.1 & I & I & I & I \\
\hline \multirow{3}{*}{7500} & 0.6 & $(0.899,0.588)$ & -596.8 & I & / & I & I \\
\hline & 1.2 & $(0.850,0.333)$ & -263.3 & I & I & I & I \\
\hline & 1.8 & $(0.410,0.602)$ & -232.0 & I & I & I & 1 \\
\hline
\end{tabular}

Table 14: The minimum vorticity $\omega_{\min }$ at negative vortex in a oblique wall-dipole collision and its location at $t=0.6,1.2,1.8$.

To test the convergence of the oblique dipole wall collision, we calculate the L2- error for the vorticity and the energy at two times. The relative error when $R e=625$ is computed with respect to the results with the finest resolution, in our case is $N_{l b}=3073$. The L2- error are defined as

$$
\text { error }=\sqrt{\frac{\sum_{i, j}\left(\omega_{i j}\left(N_{l b}\right)-\omega_{i j}\left(N_{l b \max }\right)\right)^{2}}{\sum_{i, j}\left(\omega_{i j}\left(N_{l b \max }\right)\right)^{2}}} .
$$

Since the behaviour of the dipole change by the dipole colliding with the wall, we attempted to calculate the error at different times. In Figure 20 we plot the errors $E(t)$ and $\omega$ at $t=0.3$ and $t=0.5$ for different resolutions where the second order error are shown for TRT-LBM as expected. 


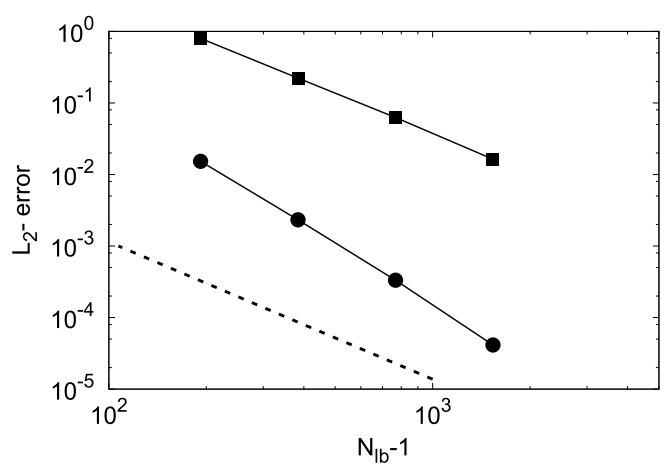

(a) $t=0.3$

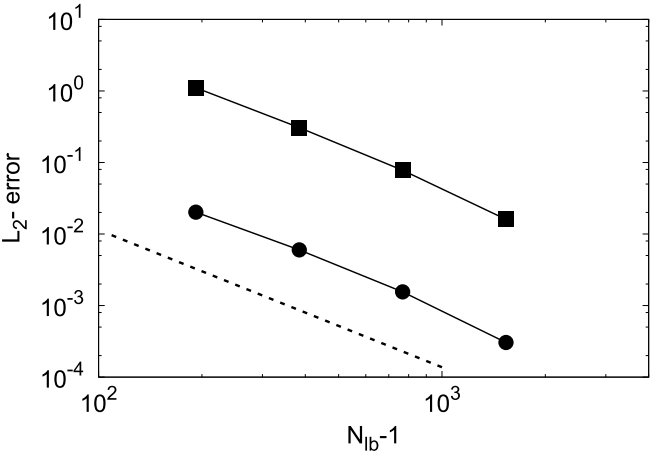

(b) $t=0.5$

Figure 20: The $L_{2}$ - error for the oblique dipole wall collision for the vorticity (fill square) and the total kinetic energy (fill circle). The error for $R e=625$ at $t=0.3$ (left) and $t=0.5$ (right). The line of slop 2 (dashed) is also shown.

\section{Conclusion}

In this work we performed a detailed numerical study of moment-based boundary conditions for lattice Boltzmann simulations with no-slip walls. This method imposed boundary conditions on the hydrodynamic moments and then translated these into conditions for the unknown distribution functions $\bar{f}_{k}$ of the LBE at the walls. We have shown that the LBM supplemented with moment based boundary conditions is an accurate numerical method that is competitive compared with other flow solvers. Simultaneously, we studied the physics of the dipole when it hit the no slip wall at different Reynolds numbers. For normal and oblique wall collision, the convergence of the total enstrophy and energy were investigated and the convergence of the total angular momentum was also tested for the oblique case.

At the beginning of this flow, the primary monopoles are released from the two semi circular vortices which propelled towards the right wall. The surrounding shields moved to the opposite direction to create a weaker dipole that hits the left wall to create additional weak vortices. These vortices have no significant impact on the behaviour of the flow. The interaction of the primary dipole with the no slip right wall created and induced a secondary dipole which interacted with the other vortices on the boundary. After the first collision with the wall the symmetry between the two monopoles remained in the normal case while it was broken in the oblique case. For both cases, The energy dissipation rate in general decreased when the Reynolds number increased. As the Reynolds number increased, the ratio between the first and second maximum enstrophy increased.

To increase our understanding of dipole wall collisions further we investigated the behaviour of the vortices at the boundary at different times. In the normal case, small and high value vortices were created at the wall that merged with the primary vortex at higher Reynolds numbers. For the oblique case, third and fourth small monopoles were created from the boundary layer wall and become more obvious as the Reynolds number increased. Moreover, for both cases we showed the behaviour of the vortices at $x=1$ for different times and Reynolds numbers and we found that the $\omega(t)$ at Reynolds higher than 625 have increased extra after the second dipole wall collision. Moreover, the maximum vorticity was decreased with respect with time except for higher Reynolds number which found a variations 
in the results for oblique case.

In summary, the moment-based boundary conditions can impose the hydrodynamic no-slip condition at grid points precisely without needing ad-hoc closure approximations or constraints on non-hydrodynamic moments. It should be noted that the methodology is quite general for on-grid constraints. One may impose the physical condition required on the necessary moments, provided the moments are independent $[45,44,47]$. This is of use to many single and multiphase flows. Its shortcoming, at present, is geometric flexibility. The methodology may be extended to higher dimensional lattices, and this discussion is reserved for a future publication [52]. The suitability of the methodology to complex geometries is yet to be seen. In principle, it can be used in combination with interpolation techniques although it is suspected that this will become expensive and laborious for highly irregular geometries. Thus we argue not that one method for imposing boundary conditions on LBM algorithms is necessarily and universally superior to another, but that bounce-back and moment-based conditions are best suited in different niches.

The method presented here has been shown to compute solutions that are in very good agreement with benchmark data, including results obtained from spectral method simulations. The LBM with moment based boundary conditions is second order accurate, as verified by our convergence studies, and the model discussed here successfully predicted the complex flow in the vicinity of the corners. This increases our confidence in the application of the LBM with moment-based boundary conditions to flows in confined but regular geometries.

\section{References}

[1] P. Orlandi, Vortex dipole rebound from a wall, Phys. Fluids A: Fluid Dynamics (1989-1993) 2 (1990) 1429-1436.

[2] B. Jamart, J. Nihoul, Mesoscale/Synoptic Coherent Structures in Geophysical Turbulence, Elsevier, New York, 1989.

[3] E. Coutsias, J.-P. Lynov, Fundamental interactions of vortical structures with boundary layers in two-dimensional flows, Physica D 51 (1991) $482-497$.

[4] H. Clercx, G. van Heijst, Dissipation of kinetic energy in two-dimensional bounded flows, Phys. Rev. E 65 (2002) 066305.

[5] H. Clercx, C.-H. Bruneau, The normal and oblique collision of a dipole with a no-slip boundary, Comput. Fluids 35 (2006) $245-279$.

[6] M. Wells, H. Clercx, G. Van Heijst, Vortices in oscillating spin-up, J. Fluid Mech. 573 (2007) 339-369.

[7] W. Kramer, Dispersion of tracers in two-dimensional bounded turbulence, Ph.D. thesis, Eindhoven University of Technology, Eindhoven, Netherlands (2007)

[8] A. Cieślik, R. Akkermans, L. Kamp, H. Clercx, G. Van Heijst, Dipole-wall collision in a shallow fluid, Eur. J. Mech. B/Fluids 28 (2009) $397-404$.

[9] J. Guzmán, L. Kamp, G. Van Heijst, Vortex dipole collision with a sliding wall, Fluid Dyn. Res. 45 (2013) 045501.

[10] J. Latt, B. Chopard, A benchmark case for lattice Boltzmann: turbulent dipole-wall collision, Int. J. Mod. Phys. C 18 (2007) 619-626.

[11] J. Hardy, O. De Pazzis, Y. Pomeau, Molecular dynamics of a classical lattice gas: Transport properties and time correlation functions, Phys. Rev. A 13 (1976) 1949.

[12] L. Luo, Lattice-gas automata and lattice Boltzmann equations for two-dimensional hydrodynamics, Ph.D. thesis, Georgiargia Institute of Technology (1993).

[13] L. Luo, The lattice-gas and lattice Boltzmann methods: past, present, and future.

[14] D. Wolf-Gladrow, Lattice-gas cellular automata and lattice Boltzmann models: An Introduction, Springer Science \& Business Media, 2000.

[15] Z. Guo, C. Shu, Lattice Boltzmann method and its applications in engineering, World Scientific, 2013. 
[16] X. He, Q. Zou, L. Luo, M. Dembo, Analytic solutions of simple flows and analysis of nonslip boundary conditions for the lattice Boltzmann BGK model, J. Stat. Phys. 87 (1997) 115-136.

[17] X. He, L.-S. Luo, Theory of the lattice Boltzmann method: From the Boltzmann equation to the lattice boltzmann equation, Phys. Rev. E 56 (6) (1997) 6811.

[18] X. Shan, X. Yuan, H. Chen, Kinetic theory representation of hydrodynamics: a way beyond the Navier-Stokes equation, J. Fluid Mech. 550 (2006) 413-441.

[19] X. He, L. Luo, A priori derivation of the lattice Boltzmann equation, Phys. Rev. E 55 (1997) R6333.

[20] P. Dellar, Incompressible limits of lattice Boltzmann equations using multiple relaxation times, J. Comput. Phys. 190 (2003) $351-370$.

[21] R. Allen, T. Reis, Moment-based boundary conditions for lattice boltzmann simulations of natural convection in cavities, Prog. Comp. Fluid Dyn.: An Int. J. 16 (4) (2016) 216-231.

[22] D. d'Humiereśs, Generalized lattice-Boltzmann equations, Prog. Astronaut. Aeronaut. (1992) 450-458.

[23] P. Lallemand, L. Luo, Theory of the lattice Boltzmann method: Dispersion, dissipation, isotropy, Galilean invariance, and stability, Phys. Rev. E 61 (2000) 6546.

[24] A. Ladd, Numerical simulations of particulate suspensions via a discretized Boltzmann equation. part 1. theoretical foundation, J. Fluid Mech. 271 (1994) 285-309.

[25] T. Reis, T. Phillips, Alternative approach to the solution of the dispersion relation for a generalized lattice Boltzmann equation, Phys. Rev. E 77 (2) (2008) 026702.

[26] I. Ginzburg, Lattice Boltzmann modeling with discontinuous collision components: Hydrodynamic and advection-diffusion equations, J. Stat. Phys. 126 (1) (2007) 157-206.

[27] I. Ginzburg, Equilibrium-type and link-type lattice Boltzmann models for generic advection and anisotropic-dispersion equation, Adv. Water Resour 28 (11) (2005) 1171-1195

[28] I. Ginzburg, D. d'Humières, A. Kuzmin, Optimal stability of advection-diffusion lattice Boltzmann models with two relaxation times for positive/negative equilibrium, J. Stat. Phys. 139 (6) (2010) 1090-1143.

[29] S. Chen, G. Doolen, Lattice Boltzmann method for fluid flows, Annu. rev. Fluid Mech. 30 (1998) 329-364.

[30] I. Ginzbourg, P. Adler, Boundary flow condition analysis for the three-dimensional lattice Boltzmann model, J. Phys. II. France 4 (1994) $191-214$.

[31] I. Ginzburg, D. d‘Humiereśs, Multireflection boundary conditions for lattice Boltzmann models, Phys. Rev. E 68 (2003) 066614.

[32] Q. Zou, X. He, On pressure and velocity boundary conditions for the lattice Boltzmann BGK model, Phys. Fluids. 9 (1997) 1591-1598

[33] O. Filippova, D. Hänel, Grid refinement for lattice-bgk models, J. Comp. Phys. 147 (1) (1998) 219-228.

[34] A. Dupuis, B. Chopard, Theory and applications of an alternative lattice boltzmann grid refinement algorithm, Phys. Rev. E 67 (6) (2003) 066707.

[35] M. Bouzidi, M. Firdaouss, P. Lallemand, Momentum transfer of a boltzmann-lattice fluid with boundaries, Phys. Fluids 13 (11) (2001) 3452-3459.

[36] D. Yu, R. Mei, W. Shyy, A unified boundary treatment in lattice boltzmann method, in: 41st Aerospace Sciences Meeting and Exhibit, 2003, p. 953.

[37] W. Zhao, W. Yong, A family of single-node second-order boundary schemes for the lattice boltzmann method, arXiv preprint arXiv: 1712.08288

[38] Z. Li, J. Favier, U. D’Ortona, S. Poncet, An immersed boundary lattice Boltzmann method for single-and multi-component fluid flows, J. Comp. Phys 304 (2016) 424-440.

[39] D. Noble, S. Chen, G. Georgiadis, R. O. Buckius, A consistent hydrodynamic boundary condition for the lattice Boltzmann method, Phys. Fluids 7 (1) (1995) 203-209.

[40] I. Ginzbourg, D. d'Humières, Local second-order boundary methods for lattice boltzmann models, J. Stat. Phys. 84 (1996) $927-971$.

[41] C. Chang, C.-H. Liu, C. Lin, Boundary conditions for lattice Boltzmann simulations with complex geometry flows, Comput. Math. Appl. 58 
(2009) $940-949$.

[42] C. Ho, C. Chang, K.-H. Lin, C. Lin, Consistent boundary conditions for 2D and 3D lattice Boltzmann simulations, CMES 44 (2009) 137 155.

[43] M. Schlaffer, Non-reflecting boundary conditions for the lattice Boltzmann method, Ph.D. thesis, Technischen Universität München (2009).

[44] T. Reis, P. Dellar, Moment-based formulation of Navier-Maxwell slip boundary conditions for lattice Boltzmann simulations of rarefied flows in microchannels, Phys Fluids.

[45] S. Bennett, A lattice Boltzmann model for diffusion of binary gas mixtures, Ph.D. thesis, University of Cambridge (2010).

[46] P. Dellar, Moment-based boundary conditions for lattice Boltzmann magnetohydrodynamics, in: Numerical Mathematics and Advanced Applications 2011, Springer, 2013, pp. 83-90.

[47] A. Hantsch, T. Reis, U. Gross, Moment method boundary conditions for multiphase lattice Boltzmann simulations with partially-wetted walls, J. Comput. Multiphase Flows 7 (2015) 1-14

[48] S. Mohammed, T. Reis, Using the lid-driven cavity flow to validate moment-based boundary conditions for the lattice Boltzmann equation, Arch. Mech. Eng. 64 (1) (2017) 57-74.

[49] X. He, X. Shan, G. Doolen, Discrete Boltzmann equation model for nonideal gases, Phys. Rev. E 57 (1998) R13.

[50] J. Latt, B. Chopard, Lattice Boltzmann method with regularized non-equilibrium distribution functions, arXiv preprint physics/0506157.

[51] D. d'Humiereśs, I. Ginzburg, Viscosity independent numerical errors for lattice Boltzmann models: from recurrence equations tomagic collision numbers, Comput. Math. Appl. 58 (2009) 823-840.

[52] I. Krastins, A. Kao, K. Pericleous, T. Reis, Moment-based boundary conditions for three dimensional lattice boltzmann simulations, in preparation. 